\title{
Molecular Insight into the Regulation of Vimentin by Cysteine Modifications and Zinc Binding
}

\author{
Andreia Mónico (D), Joan Guzmán-Caldentey, María A. Pajares (D), Sonsoles Martín-Santamaría * (D) \\ and Dolores Pérez-Sala *(D)
}

check for

updates

Citation: Mónico, A.;

Guzmán-Caldentey, J.; Pajares, M.A.;

Martín-Santamaría, S.; Pérez-Sala, D.

Molecular Insight into the Regulation of Vimentin by Cysteine

Modifications and Zinc Binding.

Antioxidants 2021, 10, 1039. https://

doi.org/10.3390/antiox10071039

Academic Editors: Nikolaus Bresgen and Werner Siems

Received: 22 May 2021

Accepted: 24 June 2021

Published: 28 June 2021

Publisher's Note: MDPI stays neutral with regard to jurisdictional claims in published maps and institutional affiliations.

Copyright: (c) 2021 by the authors. Licensee MDPI, Basel, Switzerland. This article is an open access article distributed under the terms and conditions of the Creative Commons Attribution (CC BY) license (https:/ / creativecommons.org/licenses/by/ $4.0 /)$.
Department of Structural and Chemical Biology, Centro de Investigaciones Biológicas Margarita Salas (CSIC), 28040 Madrid, Spain; andreiamonico89@gmail.com (A.M.); joanguzman@gmail.com (J.G.-C.); mapajares@cib.csic.es (M.A.P.)

* Correspondence: smsantamaria@cib.csic.es (S.M.-S.); dperezsala@cib.csic.es (D.P.-S.)

Abstract: The intermediate filament protein vimentin is involved in essential cellular processes, including cell division and stress responses, as well as in the pathophysiology of cancer, pathogen infection, and autoimmunity. The vimentin network undergoes marked reorganizations in response to oxidative stress, in which modifications of vimentin single cysteine residue, Cys328, play an important role, and is modulated by zinc availability. However, the molecular basis for this regulation is not fully understood. Here, we show that Cys328 displays a low $\mathrm{pK}_{\mathrm{a}}$, supporting its reactivity, and is readily alkylated and oxidized in vitro. Moreover, combined oxidation and crosslinking assays and molecular dynamics simulations support that zinc ions interact with Cys328 in its thiolate form, whereas Glu329 and Asp331 stabilize zinc coordination. Vimentin oxidation can induce disulfide crosslinking, implying the close proximity of Cys328 from neighboring dimers in certain vimentin conformations, supported by our computational models. Notably, micromolar zinc concentrations prevent Cys328 alkylation, lipoxidation, and disulfide formation. Moreover, zinc selectively protects vimentin from crosslinking using short-spacer cysteine-reactive but not amine-reactive agents. These effects are not mimicked by magnesium, consistent with a lower number of magnesium ions hosted at the cysteine region, according to molecular dynamics simulations. Importantly, the region surrounding Cys328 is involved in interaction with several drugs targeting vimentin and is conserved in type III intermediate filaments, which include glial fibrillary acidic protein and desmin. Altogether, our results identify this region as a hot spot for zinc binding, which modulates Cys328 reactivity. Moreover, they provide a molecular standpoint for vimentin regulation through the interplay between cysteine modifications and zinc availability.

Keywords: vimentin assembly; redox interplay; lipoxidation; cysteine; zinc binding; oxidative modifications; divalent cation; crosslinking

\section{Introduction}

Vimentin is a type III intermediate filament protein that forms robust filaments in mesenchymal cells. The intermediate filament network maintains an intimate crosstalk with the other cytoskeletal systems (microfilaments and microtubules) and modulates essential cell properties, from cell size to migration, division, and elasticity [1-3]. In addition, vimentin is involved in disease by various mechanisms, including acting as a cellular receptor for pathogens, such as bacteria and viruses [4], regulating the immune response [5], or playing a key role in the epithelial-mesenchymal transition during tumorigenesis [6], for which it is considered a critical drug target $[4,7,8]$. Importantly, the vimentin network finely responds to electrophilic and oxidative stress, a function in which its single cysteine residue (Cys328) plays an important role [9,10]. Indeed, various electrophilic lipids including 4-hydroxynonenal, cyclopentenone prostaglandins, and nitrated phospholipids induce marked vimentin reorganization $[10,11]$. 
The cellular architecture of vimentin filaments is still not completely understood. In contrast to the polarity observed in microfilaments and microtubules, vimentin filaments are non-polar and can grow at both ends. Moreover, they are highly dynamic in cells and can exchange subunits along their length and undergo severing and reannealing $[12,13]$. Nevertheless, the regulation and/or cofactors modulating these processes need further characterization. Vimentin polymerization in vitro has been exhaustively analyzed by a variety of techniques, including electron microscopy (EM) and cryo-EM, atomic force microscopy, small-angle X-ray scattering, sedimentation velocity, and total internal reflection fluorescence [14-19]. A consensus regarding the assembly pathway has emerged according to which parallel vimentin homodimers engage in a staggered manner in antiparallel tetramers, eight of which laterally associate to give a unit-length filament (ULF) (Figure 1A). These ULFs assemble head to tail to form filaments. During or after this elongation process, filaments "mature", undergoing an internal rearrangement by which the initial ULF diameter ( 16 nm) diminishes down to 10-12 nm, considered the diameter of normal filaments $[14,20]$.

Vimentin monomers consist of three domains, head, rod, and tail, of which the rod is mainly alpha-helical, whereas the head and tail domains lack a defined structure. Being a coiled-coil protein for a high proportion of its sequence, the full crystal structure of vimentin has not been solved yet. Using "divide and conquer" approaches, the structure of some vimentin segments in different degrees of association, i.e., dimers or tetramers, has been obtained (please, see [21] for review). In addition, elegant early works using amine-reactive crosslinkers, such as disuccinimidyl tartrate (DST), have yielded insight into the disposition of vimentin dimers in tetramers and of tetramers within filaments [22,23]. The results obtained suggested the predominance of an arrangement involving the aminoterminal overlap of the two dimers, the so-called A11 mode; in preparations of soluble vimentin, and an increased abundance of other alignment modes coexisting in vimentin filaments, namely, a C-terminal overlap, the A22 mode; a head to tail overlap, the ACN mode; or a complete antiparallel overlap, the A12 mode. The way these associations could be present within filaments is illustrated in a cartoon view of a two-ULF assembly (Figure 1A). More recently, deuterium exchange approaches have yielded information on the vimentin head domain [24], whereas electron paramagnetic resonance has been used to obtain insight into the structure of the head [25] and tail domains [26]. Information collected through these approaches has been integrated with molecular modeling studies providing our current view of vimentin assembly [17,27] (reviewed in [28]). Moreover, information from various intermediate filament proteins has contributed to the understanding of filament structure $[29,30]$. Most available models of the vimentin tetramer adopt the A11 configuration, in which an anchoring knob-hydrophobic pocket mechanism involving the 1B segment, identified in several intermediate filament proteins including vimentin, provides stability [31] (see [30] for review). In turn, in ULF or filaments, a parallel stacking of tetramers is proposed, usually represented as a tubular structure $[17,32,33]$ (Figure 1B), although their exact disposition and relative positions with respect to the longitudinal axis are not understood.

Vimentin contains a single cysteine residue, Cys328, located in coil 2B (Figure 1C), which appears to be required for oxidative vimentin filament disruption in vitro and network rearrangement in cells, thus acting as a putative redox sensor [9,34,35]. Moreover, this region has been involved in the binding of potential antitumoral drugs targeting vimentin function $[36,37]$. However, the precise location of Cys328 residues within filaments and how their modifications impact filament organization remains unknown. Crystal structures of segments of coil 2B including Cys328 (residues 254-411) show the lateral chain of Cys328 facing outwards from the dimer [38,39], a fact that is also observed in models available through databases (e.g., P02543) [32,33]. However, in some cases, the length of the segment solved precludes the complete identification of interactions involving Cys328. 
A

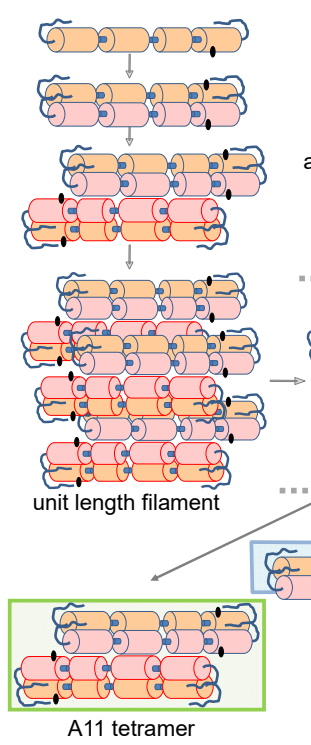

monomer

parallel dimer

antiparallel tetramer

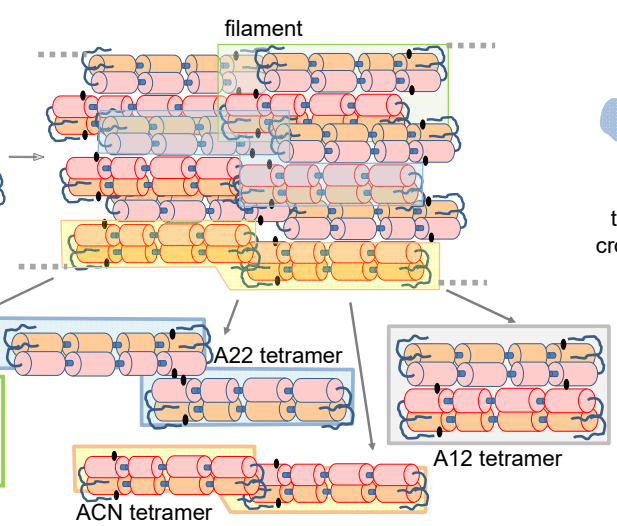

B

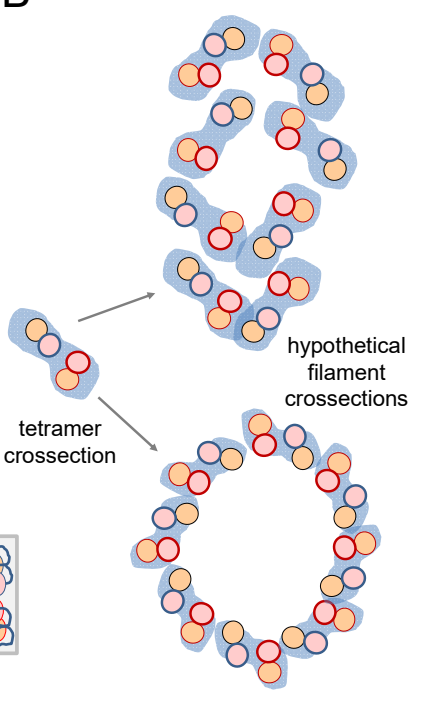

C

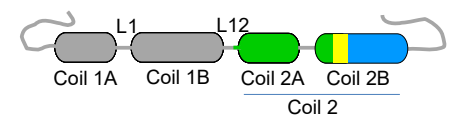

\begin{tabular}{|c|c|c|c|c|c|c|c|c|c|}
\hline 10 & 20 & 30 & 40 & 50 & 60 & 70 & 80 & 90 & 100 \\
\hline MSTRSVSSSS & YRRMFGGPGT & ASRPSSSRSY & VTTSTRTYSL & GSALRPSTSR & SLYASSPGGV & YATRSSAVRL & RSSVPGVRLL & QDSVDFSLAD & AINTEFKNTR \\
\hline 110 & 120 & 130 & 140 & 150 & 160 & 170 & 180 & 190 & 200 \\
\hline TNEKVELQEL & NDRFANYIDK & VRFLEQQNKI & LLAELEQLKG & QGKSRLGDLY & EEEMRELRRQ & VDQLTNDKAR & VEVERDNLAE & DIMRLREKLQ & EEMLQREEAE \\
\hline 210 & 220 & 230 & 240 & 250 & 260 & 270 & 280 & 290 & 300 \\
\hline NTLQSFRQDV & DNASLARLDL & ERKVESLQEE & IAFLKKLHEE & EIQELQAQIQ & EQHVQIDVDV & SKPDLTAALR & DVRQQYESVA & AKNLQEAEEW & YKSKFADLSE \\
\hline 310 & 320 & 330 & 340 & 350 & 360 & $\quad 370$ & 380 & 390 & 400 \\
\hline AANRNNDALR & QAKQESTEYR & RQVQSLTCEV & DALKGTNESL & ERQMREMEEN & EAANYQD & TIGRLQDEIQ & NMKEEMARHL & REYQDLLNVK & MALDIEIATY \\
\hline 410 & 420 & 430 & 440 & 450 & 460 & & & & \\
\hline
\end{tabular}

RKLIEGEESR ISLPLPNFSS LNLRETNLDS LPLVDTHSKR TLLIKTVETR DGQVINETSQ HHDDLE

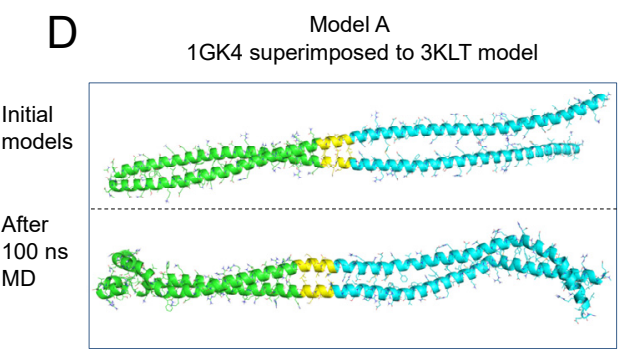

3KLT superimposed to $1 \mathrm{GK} 4$ model

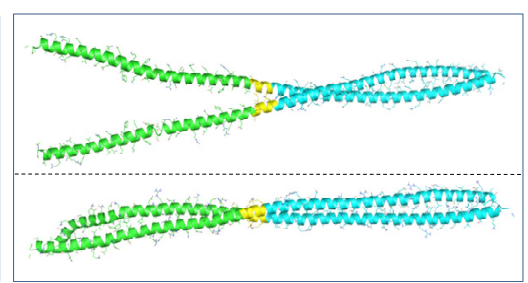

Figure 1. (A) Scheme of vimentin assembly and the proposed configurations of vimentin tetramers in filaments (please see text for details). A cartoon view interpretation of the assembly of two unit length filaments (ULF) is presented (labeled as "filament") showing the potentially key position of the cysteine residue (black dots), which could fall close to the region involved in the connection of two ULFs. Within assembled filaments, vimentin dimers can coincide in different relative positions, thus resulting in several tetrameric configurations, some of which are outlined in the figure by color shadowing and extracted below for easier visualization. (B) Cartoon of a tetramer transversal view and hypothetical dispositions of tetramers in a transversal section of a filament. Please note that these schemes are inspired in previous works [17,32] and are not based on molecular dynamics simulations. (C) Scheme of a vimentin monomer showing the distribution in coiled coil segments and linkers according to [22]. The segments used for building the models shown in (D) are highlighted by a color code: amino acids present in the 3KLT and 1GK4 crystal structures are represented in green and blue, respectively; the region shared between both crystal structures is highlighted in yellow; and the cysteine residue is shown in red. The position of those segments in the full vimentin sequence is depicted underneath by the same color code. In addition, color boxes outline the amino acids integrating the linker (blue) and the position of the stutter (purple). (D) Representation of model A and model B, built from the superimposition of 3KLT (green) and 1GK4 (blue) crystal structures on the shared region of both crystal structures (yellow). The upper pictures represent the initial model and lower pictures the model after $100 \mathrm{~ns}$ of MD simulation. 
Additional data on coil 2B interactions obtained by site-directed spin labeling of residues around Cys328 and electron paramagnetic resonance [40] reveal residue 348 as the strongest point of interaction and overlap (within $1.6 \mathrm{~nm}$ ) between two antiparallel dimers. Moreover, vimentin, as well as other intermediate filament proteins, has been reported to undergo oxidative crosslinking through its single cysteine residue [41]. The major product of this crosslinking is a disulfide-bonded oligomer, which suggests that in certain conformations of soluble or filamentous vimentin, cysteine residues, likely from different dimers or tetramers, are located at a close distance. Nevertheless, the correspondence of these conformations with the previously described modes of alignment is not straightforward.

The vimentin molecule behaves as a polyelectrolyte due to the abundance of negatively charged residues. This feature promotes its interaction with divalent cations, such as calcium and magnesium, which, at millimolar levels, influence vimentin in vitro assembly and have been reported to behave as crosslinkers for certain regions of the protein [42,43], affecting the properties of the network [44]. In recent works, we observed that zinc availability influenced the organization of the vimentin network and its resistance to oxidants in cells $[9,10]$ and that micromolar zinc concentrations modulated vimentin in vitro assembly [45]. Thus, we proposed that zinc could interact with vimentin at several sites, of which the region surrounding Cys328 could be especially relevant [46]. Zinc(II) ions interact with proteins playing catalytic, regulatory, or structural roles, usually coordinating with amino acids such as cysteine, histidine, or carboxylic amino acids [47,48]. Moreover, the interaction of $\mathrm{Zn}$ (II) ions with cysteine residues can modulate their reactivity and impact their redox regulation [49]. Therefore, given the importance of Cys328 in vimentin assembly and response to oxidative stress, elucidating its interaction with zinc will contribute to the understanding of vimentin regulation.

Here, we report a computational model of the pivotal sequence of vimentin comprised between Asp264 and Gly406 in order to gain insight into the organization of the protein and its interaction with zinc. Moreover, we have experimentally addressed the interplay of oxidative and lipoxidative modifications, bifunctional cysteine crosslinkers, and zinc. Our results provide a hypothesis for the organization of the vimentin central domain, its putative interaction with zinc, and its involvement in the formation of dimers of dimers under oxidative stress conditions, in which the cysteine residues could be spatially close. Thus, this work provides a biochemical ground for the complex and versatile modulation of vimentin in response to changes in redox status and zinc availability, pointing to its single cysteine residue as a hot spot for interplay between these factors.

\section{Materials and Methods}

\subsection{Materials}

Dimethylsulfoxide (DMSO), monosodium and disodium phosphates, Tris, Pipes, DTT, PMSF, Zincon, diamide, and dibromobimane (DBB, spacer arm, $4.88 \AA$ [50]) were from Sigma. Tris(2-maelimidoethyl)amine, (TMEA, spacer arm, $10.3 \AA$ ) and bismaleimidohexane (BMH, spacer arm, $13.0 \AA$ A) were from Thermo Fisher Scientific. Monobromobimane (MBB, sc-214629) and (+)-Biotin-(PEO)3-iodoacetamide (biotinylated iodoacetamide, Iac-B) were purchased from Santa Cruz Biotechnology. Citric acid, sodium carbonate and bicarbonate, 2-mercaptoethanol, and EDTA were obtained from Merck. Precision Plus-Dual Color protein standards and Sypro Ruby were from BioRad. The biotinylated analog of 15deoxy- $\Delta^{12,14}$-prostaglandin $\mathrm{J}_{2}\left(15 \mathrm{~d}-\mathrm{PGJ}_{2}-\mathrm{B}\right)$ was from Cayman Chemical. No unexpected or unusually high safety hazards were encountered throughout this work.

\subsection{Computational Methods}

\subsubsection{Building of the Vimentin Models}

PyMol software [51] was used to build the 3D models from the vimentin X-ray structures available at the Protein Data Bank [52] (https://www.rcsb.org, accessed on 28 June 2021), with PDB IDs 3KLT and 1GK4. The sequences of PDBs 3KLT and 1GK4 structures share seven amino acids (Cys328, Glu329, Val330, Asp331, Ala332, Leu333, 
and Lys334). One monomer of each PDB structure was kept and superimposed onto the dimer from the other PDB structure by this overlapping region. Thus, two vimentin dimer models comprising residues Asp264 through Gly406, but differing in their geometry, were constructed: 1GK4 superimposed onto 3KLT model (Model A) and 3KLT superimposed to 1GK4 model (Model B). Only Model B was considered for further computational studies.

\subsubsection{Protein-Protein Docking}

Model B of vimentin dimer from the molecular dynamics (MD) simulations was submitted to HADDOCK [53] and ZDOCK [54] servers to perform protein-protein docking. No restrictions were applied. Docked solutions with cross shape were discarded. A total of 20 vimentin dimers of dimers were finally selected and submitted to $1 \mathrm{~ns}$ MD simulations for analysis. Only two dimer-dimer assemblies (from HADDOCK) with antiparallel orientation and an overall longitudinal alignment were finally selected.

\subsubsection{Molecular Dynamics Simulations}

MD simulations were carried out using Amber14. The system was solvated with the TIP3P water molecules model, and the simulation box was built such that the edges were at least $10 \AA$ distant from any atom. Eight steps of preparation were performed before running MDs. The first one consisted of 1000 steps of the steepest descent algorithm followed by 7000 steps of conjugate gradient algorithm; a $100 \mathrm{kcal} \cdot \mathrm{mol}^{-1} \cdot \mathrm{A}^{-2}$ harmonic potential constraint was applied on both the proteins and the ligand. In the four subsequent steps, the harmonic potential was progressively lowered, respectively, to 10, 5, and $2.5 \mathrm{kcal} \cdot \mathrm{mol}^{-1} \cdot \mathrm{A}^{-2}$ for 600 steps of conjugate gradient algorithm each time, and then the whole system was minimized uniformly. In the following step, the system was heated from 0 to $100 \mathrm{~K}$ using the Langevin thermostat in the canonical ensemble (NVT) while applying a $20 \mathrm{kcal} \cdot \mathrm{mol}^{-1} \cdot \mathrm{A}^{-2}$ harmonic potential restraint on the proteins and the ligand. The next step heated up the system from 100 to $300 \mathrm{~K}$ in the isothermal-isobaric ensemble (NPT) under the same restraint condition as in the previous step. In the last step, the same parameters were used to simulate the system for 100 ps, but no harmonic restraint was applied. At this point, the system was ready for the production run, which was performed using the Langevin thermostat under the NPT ensemble, at a $2 \mathrm{fs}$ time step. All production runs were performed for 100 or $150 \mathrm{~ns}$. Zinc atoms were added to the system using leap. Of note, no secondary structural changes were observed during MD simulations. All the studied structures (Asp264-Gly406 region) were quite stable, as can be inferred from the RMDS values (Supporting Information). Nevertheless, changes in other regions of the protein cannot be excluded.

\subsubsection{Computational Estimation of Cys328 $\mathrm{pK}_{\mathrm{a}}$}

The models of vimentin dimer $B$ and the corresponding vimentin dimer of dimers assembly were used for calculations. PROPKA [55,56] was employed to predict the $\mathrm{pK}_{\mathrm{a}}$ of Cys328 at physiological $\mathrm{pH}(\mathrm{pH}=7)$.

\subsection{Vimentin Refolding}

Recombinant human vimentin wild type (wt) and Cys328Ser in $8 \mathrm{M}$ urea, $5 \mathrm{mM}$ Tris$\mathrm{HCl}$ pH 7.6, 1 mM EDTA, 10 mM 2-mercaptoethanol, 0.4 mM PMSF, and approximately $0.2 \mathrm{M} \mathrm{KCl}$, purified essentially as described [57], were purchased from Biomedal (Spain). The proteins were ultrafiltrated using $10 \mathrm{~K}$ pore size Amicon Ultra filter units (Millipore) and step-wise dialyzed against $5 \mathrm{mM}$ Pipes-Na pH 7.0, $1 \mathrm{mM}$ DTT containing decreasing urea concentrations $(8,6$, and $2 \mathrm{M})$. Final dialysis was performed for $16 \mathrm{~h}$ at $16^{\circ} \mathrm{C}$ in $5 \mathrm{mM}$ Pipes-Na, pH 7.0, 0.25 mM DTT [58]. Ultrafiltration was necessary for efficient removal of EDTA [58]. Protein concentration was estimated from its $\mathrm{A}_{280} \mathrm{~nm}$, using an extinction coefficient of $22,450 \mathrm{M}^{-1} \mathrm{~cm}^{-1}$. Aliquots of the protein were kept at $-80^{\circ} \mathrm{C}$ until use. 


\subsection{Experimental Determination of the Cysteine $p K_{a}$}

Buffers were prepared by mixing $5 \mathrm{mM}$ concentrations of each of the following to obtain the desired $\mathrm{pH}$ : citric acid/disodium phosphate ( $\mathrm{pH} 3-5.5)$, disodium phosphate/monosodium phosphate $(\mathrm{pH} 6-8)$, Tris/HCl $(\mathrm{pH} 8.5-9)$, and sodium carbonate/sodium bicarbonate ( $\mathrm{pH}$ 9.5-10.8). Purified vimentin wt or Cys328Ser was thawed and subjected to five cycles of dilution with $5 \mathrm{mM}$ Pipes/Na pH $7.0(10-12 \mathrm{~mL})$ and ultrafiltration on Amicon Ultra filter units for DTT elimination, immediately before monobromobimane (MBB) modification. The last flowthrough was kept, and the protein concentration was measured on a Nanodrop (Thermo Fisher). MBB $50 \mathrm{mM}$ was prepared fresh in DMSO and later diluted with $5 \mathrm{mM}$ Pipes/Na pH 7.0 to $333 \mu \mathrm{M}$, the concentration required to obtain a final 1:4 $(\mathrm{mol} / \mathrm{mol})$ ratio protein:MBB in the modification protocol. MBB reactions were carried out in triplicate in the dark on black-polystyrene NBS-treated multiwell plates (ref. 3650, Corning) in a final volume of $100 \mu \mathrm{L} /$ well. Each well contained $2.5 \mu \mathrm{M}$ vimentin wt or Cys328Ser, $77 \mu \mathrm{L}$ of buffer at the desired $\mathrm{pH}$, and $10 \mu \mathrm{M}$ MBB. Controls lacking the protein included the flowthrough of ultrafiltration to establish the background or reduced glutathione (G4251, Sigma) as a positive control of MBB modification. Kinetics of modification were followed by measuring fluorescence emission at $480 \mathrm{~nm}$ every $3 \mathrm{~min}$ for up to $90 \mathrm{~min}$, upon excitation at $380 \mathrm{~nm}$ using Varioskan Flash (Thermo Fisher). The final $\mathrm{pH}$ for each reaction was measured with a $\mathrm{pH}$-meter using equivalent mixtures in a final volume of $2 \mathrm{~mL}$. Fluorescence data for each well were adjusted to a sigmoidal curve using GraphPad Prism v.5 to obtain the value of the bottom plateau corresponding to the initial non-alkylated protein $\left(\mathrm{F}_{\mathrm{o}}\right)$. The ratio of alkylated protein at time $t\left(\mathrm{~F}_{\mathrm{t}}\right)$ vs. $\mathrm{F}_{\mathrm{o}}$ was calculated and used to obtain the $\ln \left(\mathrm{F}_{t} / \mathrm{F}_{\mathrm{o}}\right)$. The slopes of the representation of $\ln \left(\mathrm{F}_{\mathrm{t}} / \mathrm{F}_{\mathrm{o}}\right)$ against time provide the $\mathrm{k}_{\mathrm{obs}}$ values, which were represented against the final reaction $\mathrm{pH}$ to obtain the $\mathrm{pK}_{\mathrm{a}}$ (the inflexion point of the curve). Three independent experiments were carried out for both vimentin wt and Cys328Ser using different protein preparations.

Additional verification of the results obtained with MBB was achieved by subjecting vimentin to modification with Iac-B at different $\mathrm{pHs}$ for $30 \mathrm{~min}$ at room temperature, in the dark. Reactions contained $2.5 \mu \mathrm{M}$ vimentin wt or Cys328Ser, $50 \mu \mathrm{M}$ Iac-B, and buffers at different $\mathrm{pHs}$ near the $\mathrm{pK}_{\mathrm{a}}$ values calculated in the MBB modification assays. Reactions were stopped by boiling in Laemmli sample buffer. Proteins were separated in 10\% SDSpolyacrylamide gels and electrotransferred to PVDF membranes using a semi-dry system (Bio-Rad). Biotin incorporation was assessed by incubating blots with streptavidin-HRP (1:1000 $v / v$, GE Healthcare) and vimentin levels estimated by western blot with antivimentin V9 monoclonal antibody (1:1000 $v / v$; sc-6260, Santa Cruz), followed by enhanced chemiluminescent (ECL) detection (GE Healthcare). Signal intensities were evaluated by image scanning and analysis with ImageJ.

\subsection{Vimentin Modification by Oxidants, Electrophiles, and Crosslinkers}

Modification of vimentin was assessed by gel-based techniques. Briefly, vimentin at $3.8 \mu \mathrm{M}$ in $5 \mathrm{mM}$ Pipes-Na, $\mathrm{pH}$ 7.0, was incubated for $1 \mathrm{~h}$ at room temperature in the presence of the indicated compounds. Given the fact that DTT can react with electrophilic compounds and chelate metals [59], its final concentration was kept below $0.2 \mathrm{mM}$. To evaluate the protective effect of the different salts on vimentin modification or crosslinking, the protein was preincubated with $\mathrm{ZnCl}_{2}, \mathrm{MgCl}_{2}$, or $\mathrm{NaCl}$ for $1 \mathrm{~h}$ at room temperature, as indicated. Incorporation of $15 \mathrm{~d}-\mathrm{PGJ}_{2}-\mathrm{B}$ or Iac-B was analyzed by SDS-PAGE followed by blot and biotin detection as described above. For detection of crosslinked products, samples were run on $7.5 \%$ SDS-polyacrylamide gels, which were fixed with $40 \%(v / v)$ methanol and $10 \%(v / v)$ acetic acid for 60 min, stained with Sypro Ruby overnight, washed with $10 \%(v / v)$ methanol and $6 \%(v / v)$ acetic acid, and visualized under UV light on a Gel-Doc XR Imaging System (Bio-Rad). 


\subsection{Competition Assay with Zincon}

The ability of vimentin to compete for zinc binding in the presence of Zincon was assessed by a modification of a previously reported method [60]. Immediately before the assay vimentin in $5 \mathrm{mM}$ Pipes $\mathrm{pH}$ 7.0, $0.25 \mathrm{mM}$ DTT was diluted with $5 \mathrm{mM}$ Pipes, $\mathrm{pH}$ 7.5, and ultrafiltrated as above to reach a 400 -fold dilution of DTT concentration. Typical assay mixtures contained $40 \mu \mathrm{M}$ Zincon, $5 \mu \mathrm{M}$ ultrafiltrated vimentin, and variable concentrations of $\mathrm{ZnCl}_{2}$. The last filtrate was used as control. Absorbance at $620 \mathrm{~nm}$ was measured as an index of the formation of the Zincon- $\mathrm{Zn}^{2+}$ complex.

\subsection{Statistical Analysis}

All experiments were performed at least three times using different protein batches. Results are presented as average values \pm standard error of mean (SEM). Statistical analyses were carried out using GraphPad Prism v5. For comparisons of different sets of values, the Student's $t$-test was used. Differences were considered significant for $p<0.05$.

\section{Results and Discussion}

\subsection{Computational Modeling of a Vimentin Dimer and Its Interaction with Zinc}

Identification of zinc binding proteins based on sequence analysis and database searching is often unsuccessful. Indeed, vimentin is not identified using these resources. Moreover, analysis of zinc binding to vimentin by some techniques, including mass spectrometry, has proven not viable due to the ability of zinc to induce oligomeric forms of the protein and compromise its solubility. To overcome these difficulties, we undertook a computational approach. Zinc binding sites in proteins often involve cysteine residues or carboxylic amino acids [61,62]. Based on our previous work [9], we focused our attention into the Asp264-Gly406 region of vimentin surrounding the cysteine residue in order to explore its potential interaction with zinc (Figure 1C). Two different models of vimentin dimer, namely models A and B, were computationally built from the X-ray structures of PDBs 3KLT [39] and 1GK4 (Figure 1D, see Materials and Methods for details) and submitted to $100 \mathrm{~ns}$ molecular dynamics (MD) simulation (Figure 1D and Figure S1). Interestingly, both models adopted a typical coiled-coil configuration during the simulation time. However, the final structure of Model A cannot be considered as a coiled-coil, since both ends of the protein came into contact. In contrast, Model B achieved the desired coiled-coil structure after the $100 \mathrm{~ns}$ simulation (Figure 1D) and remained stable during the whole simulation time when it was extended until $200 \mathrm{~ns}$. Interestingly, the dimerization interface between monomers in this model is mostly composed of hydrophobic residues, similarly to other related dimers, such as that of vimentin rod 1B (PDB 3UF1) [63]. Of note, in this simulation, the stutter, located at residue 351 , in which the coiled motif has been reported to be disrupted, adopts a parallel distribution, not a coiled coil motif. This is consistent with previous observations [38] (reviewed in [64,65]). Moreover, the region corresponding to PDB 3KLT is maintained very similar to the reported X-ray structure, without showing relevant structural changes.

Therefore, Model B was used to interrogate the hypothesis of a potential interaction of zinc atoms in this region (Figure 2A). Firstly, mapping the electrostatic potential surface of this dimer allowed the location of 23 possible coordination sites (hot spots) for zinc ions, i.e., regions with predominant negative density charge (Figure 2A). To saturate the system, we introduced additional ions (up to 50) around the vimentin dimer. Zinc ions were manually relocated in order to fill all the binding hot spots and the systems submitted to $30 \mathrm{~ns}$ of MD simulation. Thirteen of the 50 zinc atoms remained in close contact with vimentin residues for more than $90 \%$ of the simulation time, indicating a good stability of zinc-vimentin complexes (Figure S2). All of these zinc ions were interacting with side chains from Asp or Glu residues exposed to the solvent. Of note, in some cases, zinc atoms could interact with more than one residue located either on the same chain or on different chains (see below). 


\section{A}
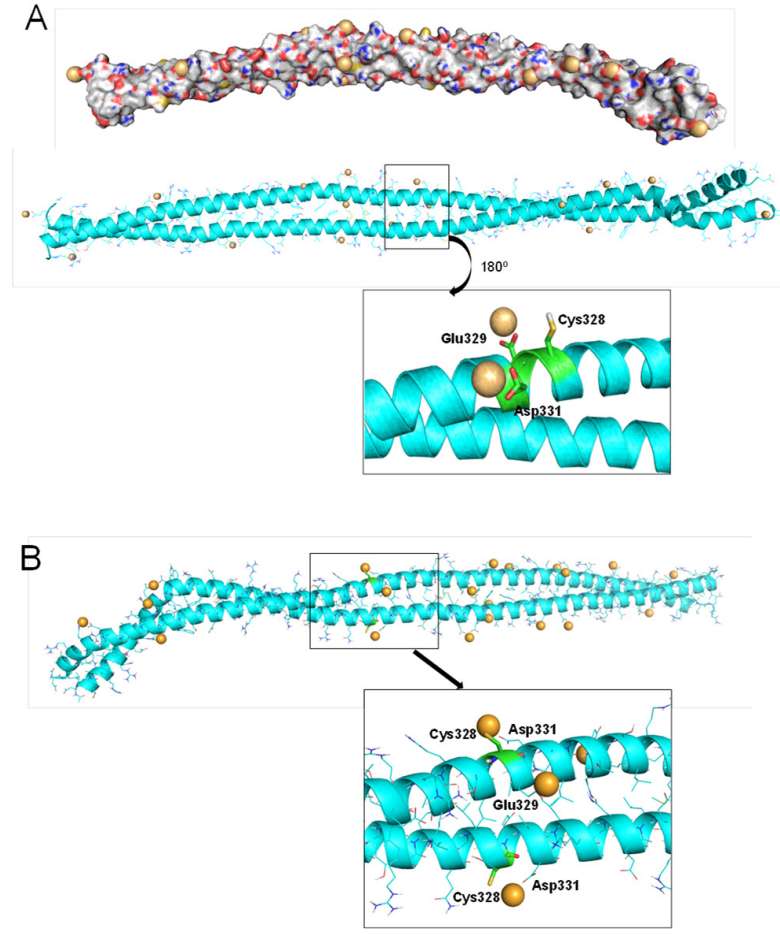

C
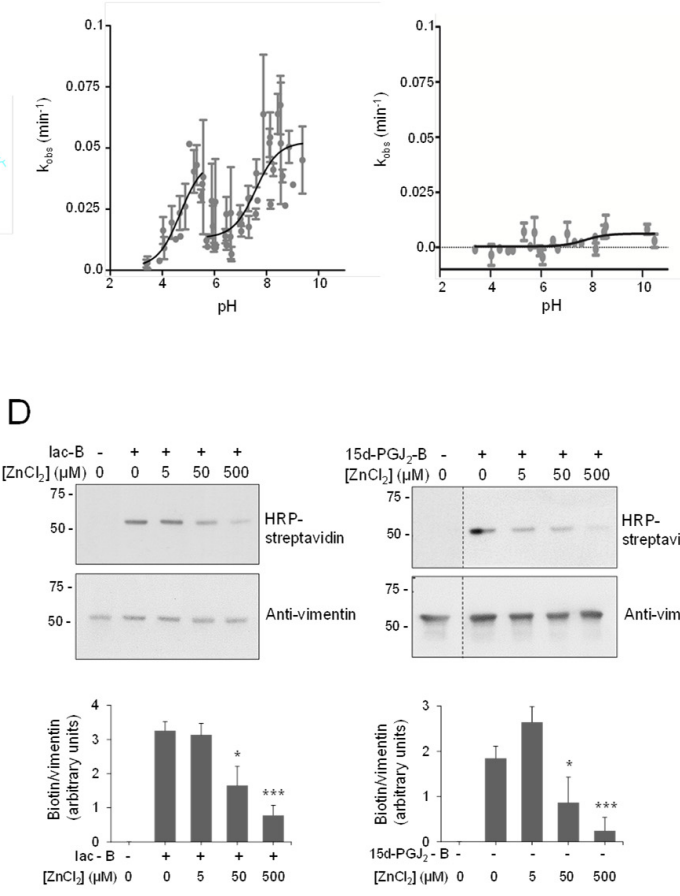
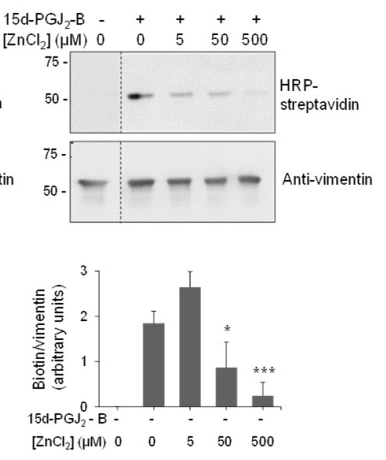

Figure 2. Vimentin dimer and its interaction with zinc. (A) Zinc binding at vimentin B dimer. Filled and ribbon views of dimer B are shown. After 100 ns MD simulation, zinc atoms, shown as orange spheres, were placed in electronegative regions. Below, the region near Cys328 is displayed in detail, illustrating the interaction of zinc atoms with Glu329 and Asp331. After $30 \mathrm{~ns}$ of MD simulation, two zinc atoms remained close to Cys328, interacting with Glu329 and Asp331 during the whole simulation time. (B) Zinc binding at vimentin B dimer, with Cys328 in its thiolate form. In this case, the cysteine interacts directly with zinc atoms, as displayed in the detailed view. (C) Experimental determination of vimentin Cys328 $\mathrm{pK}_{\mathrm{a}}$. Kinetics of MBB modification of vimentin wild type (wt, left panel) and Cys328Ser mutant (right panel) were measured at different $\mathrm{pHs}$ in triplicate using a Varioskan plate reader and the $\mathrm{k}_{\mathrm{obs}}$ calculated for each case. The $\mathrm{pK}_{\mathrm{a}}$ was obtained from the representation of $\mathrm{k}_{\mathrm{obs}}$ values against the final $\mathrm{pH}$ of the reaction. (D) Protection of vimentin modification by zinc. Purified vimentin wt ( $4.3 \mu \mathrm{M}$ final concentration) was incubated with $\mathrm{ZnCl}_{2}$ at the indicated concentrations for $1 \mathrm{~h}$ at r.t., before adding vehicle, biotinylated iodoacetamide $\left(10 \mu \mathrm{M}\right.$, Iac-B, left panel), or biotinylated 15d-PGJ $2\left(1 \mu \mathrm{M}, 15 \mathrm{~d}-\mathrm{PGJ} \mathrm{J}_{2}-\mathrm{B}\right.$, right panel), and incubating an additional hour at $37^{\circ} \mathrm{C}$. Incubation mixtures were run on SDS-PAGE gels, electro-blotted, and incorporation of the biotinylated compounds assessed by biotin detection with HRP-conjugated streptavidin. Vimentin was detected by western blot. Dotted lines indicate where lanes from the same gel have been cropped. The position of molecular weight standards (in $\mathrm{kDa}$ ) is indicated on the left. The ratio of the biotin and the vimentin signals is displayed in the graph as mean values \pm SEM of four independent assays. ${ }^{*} p<0.05$ vs. Iac-B or $15 \mathrm{~d}-\mathrm{PGJ}_{2}-\mathrm{B}$ at $0 \mu \mathrm{M} \mathrm{ZnCl}_{2} ;{ }^{* * *} p<0.0005$ vs. Iac-B or $15 \mathrm{~d}-\mathrm{PGJ}_{2}-\mathrm{B}$ at $0 \mu \mathrm{M} \mathrm{ZnCl} 2$ by Student's $t$-test.

Other proteins have been found to interact with multiple zinc atoms, mainly through carboxylic residues, which can play a structural role stabilizing subunit interactions [66]. Interestingly, two of the zinc ions bound to the Asp264-Gly406 region of vimentin were found to establish stable interactions with Glu329 and Asp331, which are located next to Cys328 (Figure 2A, inset). These stable interactions may pinpoint an important zinc coordination site that could contribute to Cys328 protection against modification by alkylating agents [9].

Importantly, cysteine residues can exist either in thiol (-SH) or thiolate $\left(-\mathrm{S}^{-}\right)$forms depending on their $\mathrm{pK}_{\mathrm{a}}$ and the environmental $\mathrm{pH}$. In our initial Model $\mathrm{B}$, we considered the neutral form of Cys328 thiol group (Figure 2A). Therefore, we next modeled vimentin with the cysteine residue in its thiolate form (Figure 2B and Figure S3). MD simulations were performed following the same protocol as for vimentin with Cys328 in its thiol form $(-\mathrm{SH})$. Interestingly, almost the same number of zinc ions remained close to the vimentin dimer along the simulation time. Additionally, two zinc atoms were located at the hot 
spots involving Glu329 and Asp331; however, in this case, the Cys328 thiolate entered into the zinc coordination shell maintaining more stable interactions (length of the $\mathrm{Zn}$ S coordination bond $2.5 \AA$, Figure $2 \mathrm{~B}$ and Figure S3). These observations point to the complexity of the vimentin-zinc interactions and the relevance of the electron density of the Cys for zinc coordination. On one hand, multiple vimentin species may coexist depending on the oligomeric state of the protein and on the ionization status of the cysteine residue. On the other hand, given the importance of Cys328 in vimentin assembly and redox sensing $[9,10]$, the interaction could have implications for the reactivity and/or accessibility of this residue, thus affecting its oxidation or modification by electrophiles.

\subsection{Determination of Cys328 $p K_{a}$}

Vimentin Cys328 has been shown to be the target of several posttranslational modifications, including glutathionylation, lipoxidation, and nitrosylation $[10,67,68]$, which typically affect cysteine residues with a low $\mathrm{pK}_{\mathrm{a}}$ that are therefore present in their thiolate form in substantial proportions at physiological $\mathrm{pH}$. The thiolate form is more reactive than the thiol, and therefore cysteine residues with low $\mathrm{pK}_{\mathrm{a}}$ are considered to be particularly reactive towards electrophilic compounds and/or more prone to oxidation. In order to assess the relative abundance of the thiol and thiolate forms of Cys328 under conditions close to physiological $\mathrm{pH}$, we set out to determine its $\mathrm{pK}_{\mathrm{a}}$. The computational calculation yielded $\mathrm{pK}_{\mathrm{a}}$ values of 9.3 for cysteines in the dimer, whereas for the dimer of dimers (see below), values of 9.3 and of 10.0 were obtained for cysteines facing outwards and towards the interdimer space, respectively (Figure S4). These values are in accordance with those recently obtained for Cys328 in the context of the 327-329 or the 321-334 vimentin peptides, for which $\mathrm{pK}_{\mathrm{a}}$ values of $8.5 \pm 2.2$ were obtained following different computational approaches [69]. Since theoretical and experimental $\mathrm{pK}_{\mathrm{a}}$ values often differ [70], we undertook the experimental determination of Cys328 $\mathrm{pK}_{\mathrm{a}}$ (Figure $2 \mathrm{C}$ ). For this purpose, we set up a procedure based on the modification of cysteine residues by the fluorescent compound monobromobimane (MBB) [71]. Incubation of soluble vimentin with MBB led to the $\mathrm{pH}$-dependent incorporation of the fluorescent probe, as shown upon calculation of $\mathrm{k}_{\mathrm{obs}}$ values for the reactions carried out at each $\mathrm{pH}$ (Figure 2C). Interestingly, the results obtained were consistent with the existence of two ionization steps for Cys328 in soluble vimentin, rendering $\mathrm{pK}_{\mathrm{a}}$ values of $4.61 \pm 0.36$ and $7.39 \pm 0.41$ (mean $\pm \mathrm{SD}$ ) (Figure 2C, left). Therefore, the experimental $\mathrm{pK}_{\mathrm{a}}$ values are much lower than the theoretical values obtained from the model or from cysteine in solution and indicate that a substantial proportion of Cys328 exists in its thiolate form at physiological $\mathrm{pH}$. Incorporation of $\mathrm{MBB}$ into Cys328 is specific, as a Cys328Ser mutant, used as a control, showed only background modification and nearly null $\mathrm{pH}$ dependence, evidenced by the dispersion of the data and the low $\mathrm{R}^{2}$ of the curve fit $\left(\mathrm{R}^{2}=0.07249\right)$ (Figure $2 \mathrm{C}$, right). The $\mathrm{pK}_{\mathrm{a}}$ of cysteine residues is greatly influenced by the chemical environment, including the proximity of charged residues or hydrogen bonding [70]. Cysteines with particularly low $\mathrm{pK}_{\mathrm{a}}$ values have been reported at the active sites of the thioredoxin superfamily [72] or of cysteine proteases [73]. However, the current available vimentin structures do not provide a complete view of the environment surrounding the lateral chain of Cys328 to discern the cause of the low $\mathrm{pK}_{\mathrm{a}}$ values detected. Moreover, the observation of two different $\mathrm{pK}_{\mathrm{a}}$ values for vimentin could be related to the presence of different oligomeric or conformational species in the protein preparations. It is well known that soluble vimentin can exist in several oligomeric species, which may include tetramers and octamers [14,23,74]. Therefore, our results could be interpreted on the basis of the coexistence of a variety of oligomeric or conformational species in soluble vimentin preparations, in which the environment of Cys328 could be significantly different, affecting its $\mathrm{pK}_{\mathrm{a}}$. Indeed, the $\mathrm{pK}_{\mathrm{a}}$ values of certain cysteine residues are highly influenced by the oligomeric state of the protein [75]. In order to strengthen these observations, the alkylation of vimentin by biotinylated iodoacetamide (Iac-B) was assessed at $\mathrm{pH}$ ranges around the $\mathrm{pK}_{\mathrm{a}}$ values determined by MBB using a gel-based assay 
(Figure S4). This semiquantitative assay confirmed the existence of two $\mathrm{pH}$ ranges for Cys328 modification, with more intense Iac-B incorporation for thiolates with $\mathrm{pK}_{\mathrm{a}}$ 7.39.

Taken together, these results indicate that the $\mathrm{pK}_{\mathrm{a}}$ of Cys328 falls within the physiological $\mathrm{pH}$ range, suggesting that a significant proportion of cysteine residues in vimentin molecules will occur in thiolate form in cells and supporting the behavior of this residue as a hot spot for oxidative modification and therefore redox sensing $[9,76]$.

\subsection{Zinc Protects Vimentin from Cysteine Alkylation and Lipoxidation}

To substantiate the importance of the interaction of zinc with the Cys 328 region, we assessed the extent of modification by Iac-B after preincubating the protein with $\mathrm{ZnCl}_{2}$ (Figure 2D). Micromolar concentrations of $\mathrm{ZnCl}_{2}$ prevented subsequent vimentin alkylation by Iac-B in a concentration-dependent manner (Figure 2D, left panel). Furthermore, $\mathrm{ZnCl}_{2}$ protected vimentin from lipoxidation by the electrophilic prostaglandin $15 \mathrm{~d}-\mathrm{PGJ} \mathrm{J}_{2}$ (Figure 2D, right panel), which selectively modifies Cys328 [10]. Protection of cysteine residues from alkylation has been previously proposed as an indication of zinc binding [77]. Thus, these results support the interaction of zinc with vimentin in the proximity of the cysteine residue. Nevertheless, the possibility that zinc could induce vimentin aggregation and/or conformational changes, reducing the accessibility of Cys328 to modification, should also be considered [45]. In contrast, we have previously reported that $\mathrm{NaCl}$-induced polymerization does not preclude vimentin oxidation or lipoxidation, which indicates that the protection is not merely due to lower cysteine accessibility in oligomeric structures and suggests a distinct role for zinc [10]. Interestingly, $\mathrm{ZnCl}_{2}$ concentrations providing protection against Cys328 modifications in our assays are in the low micromolar range, that is, in the order of the total concentrations found in plasma [78] or in cells [79], i.e., approximately 12 and $200 \mu \mathrm{M}$, respectively. Nevertheless, since these assays contain DTT, which can chelate metals [59], zinc can probably interact with vimentin at lower concentrations. Indeed, competition assays using vimentin after DTT removal by ultrafiltration and the chromophoric zinc probe Zincon ( $K_{D}$ for zinc $214 \mathrm{nM}$ ) [60] indicate that low micromolar concentrations of vimentin effectively sequester zinc (Figure S5). In cells, most zinc is bound to proteins, and its levels vary greatly depending on the subcellular compartment. Thus, picomolar to nanomolar concentrations of free zinc have been measured in the cytoplasm, whereas in certain organelles such as lysosomes or insulin-storing granules, zinc accumulates, reaching concentrations between 1 and $100 \mu \mathrm{M}[80,81]$. Therefore, the modulation of vimentin modifications by zinc in the cell may change notably depending on the local ion concentrations. From the chemical point of view, a complex interplay may exist between zinc binding and cysteine reactivity. On one hand, zinc binding can lower the $\mathrm{pK}_{\mathrm{a}}$ of cysteine residues, stabilizing the negatively charged thiolate anions and making them more reactive towards oxidants and electrophiles $[48,82]$. In any case, a low $\mathrm{pK}_{\mathrm{a}}$ implies a higher fraction of reactive thiolates, but it also may imply a lower nucleophilicity [83]. Therefore, the impact of zinc binding on thiol accessibility and reactivity needs to be evaluated on an individual basis. Indeed, there are examples of protective effects of zinc binding on cysteine modification, e.g., alkylation by iodoacetamide or oxidation $[77,84]$, but also cases in which it promotes cysteine modification, e.g., persulfidation by $\mathrm{H}_{2} \mathrm{~S}$ [84]. This interplay can be even more complex in cells, where prooxidant and antioxidant actions of zinc can take place depending on the cellular context and zinc levels [49].

\subsection{Modeling of a Vimentin Cys328Ser Mutant}

Mutation of cysteine to serine in proteins is a widely used strategy to assess the importance of thiol moieties in protein function. Regarding vimentin, we have previously shown that a Cys328Ser mutant is competent for filament formation in vitro and confers resistance to the alterations of vimentin filaments induced by oxidants and electrophilic agents [10], although it shows subtle but significant differences in polymerization features and response to zinc supplementation compared to vimentin wt [45]. Therefore, it was of interest to model a potential interaction of this mutant with zinc. The starting geometry of vimentin 
dimer B was used to computationally mutate Cys328 to serine (Figure 3). The resulting structure was submitted to $50 \mathrm{~ns}$ of MD simulation, and no major differences with respect to the wt overall structure were observed (Figure S6). However, detailed observation of the Ser328 region showed that the H-bond linking the Ser $328 \mathrm{OH}$ group and the $\mathrm{CO}$ group from the $\mathrm{Gln} 324$ backbone was weaker than the $\mathrm{H}$-bond established between the $\mathrm{SH}$ and $\mathrm{CO}$ groups in the wt protein, as deduced from monitoring the H-bond distance. Additionally, the electrostatic potential of the surface of the Cys328Ser dimer B was slightly more electronegative than that of the wt dimer. The main differences affected the zinc binding sites. Whereas the wt was shown above to bind two zinc ions, four zinc ions remained bound at the vicinity of the serine residues (two ions per residue) in the simulation of the mutant protein. Therefore, the vimentin Cys328Ser mutation confers resistance to disruption by oxidants $[9,10]$ and may also result in differences in the response to zinc availability.

A

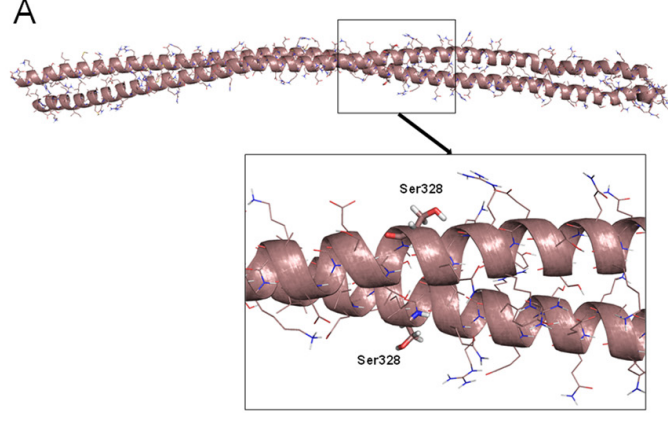

B

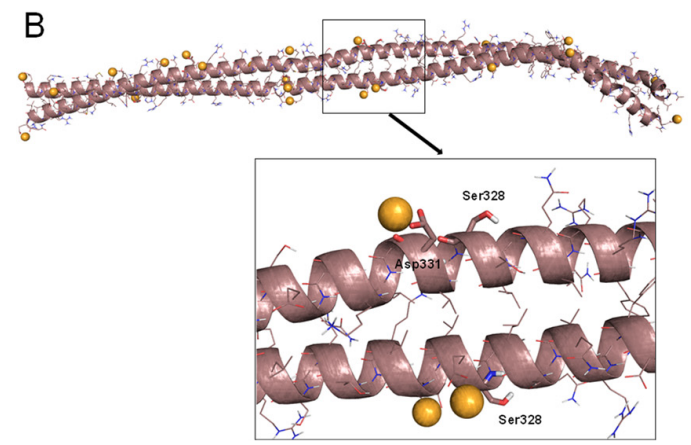

Figure 3. Dimer B of a vimentin Cys328Ser mutant and its interaction with zinc. (A) Final snapshot of a $100 \mathrm{~ns}$ MD simulation of the Cys328Ser mutant in water. (B) Final snapshot of $100 \mathrm{~ns}$ MD simulation of this mutant in the presence of zinc. In both cases, lower panels show an amplified view of the region around Ser328. Zinc atoms are represented as orange spheres.

\subsection{Modeling of a Vimentin Dimer of Dimers and Its Interaction with Zinc}

The relative position of vimentin tetramers within filaments is not completely understood yet. Importantly, oxidative conditions have been reported to induce the formation of oligomeric species of vimentin by disulfide bonding $[10,41]$, which correlates with altered assembly. Indeed, as shown in Figure 4A, incubation of vimentin with various oxidants or cysteine-crosslinking agents, such as diamide, hydrogen peroxide, or dibromobimane (DBB, spacer arm $4.88 \AA$ ), gave rise to oligomeric species migrating slightly above $150 \mathrm{kDa}$ in non-reducing SDS-PAGE.

This electrophoretical mobility, although lower than theoretically expected, is compatible with the position of previously reported vimentin dimeric species [10,41]. Of note, given the outwards orientation of cysteine residues in dimers (Figure 1A), the $150 \mathrm{kDa}$ band likely represents two covalently linked monomers originally belonging to adjacent dimers or tetramers that dissociate in SDS-PAGE. Of the various agents used, diamide and $\mathrm{H}_{2} \mathrm{O}_{2}$ induce disulfide bonds, and therefore reversible dimerization [10], whereas DBB induces irreversible crosslinking [85]. Formation of the electrophoretic vimentin dimer requires the cysteine residue, as illustrated by lack of DBB crosslinking in the Cys328Ser mutant (Figure 4B). Consistent with previous results [9], preincubation of vimentin with $\mathrm{NaCl}$ under conditions leading to filament polymerization increased the formation of the DBB-induced oligomer (Figure 4C). Taken together, these results suggest the existence of certain conformations in soluble vimentin and within filaments in which cysteine residues, likely from different tetramers, are located at a close distance. Importantly, cysteinecrosslinked oligomers also occur in other type III intermediate filament proteins, like glial fibrillary acidic protein (GFAP), which is highly homologous to vimentin [34]. Moreover, cysteine-crosslinked heterooligomers of vimentin and GFAP or desmin have been detected both in vitro and in cells, which supports the proximity of the cysteine residues in pathophysiological cytoskeletal arrangements $[34,86,87]$. Among the potential tetrameric 
associations, the A22 overlap mode (Figure 1A) would allow the shortest distance between cysteine residues in adjacent dimers, although still not ideal for disulfide formation [41]. However, as pointed out in [41], in this conformation, the stutter falls between the two cysteine residues and may impose alterations in the expected configuration in this region. Indeed, the stutter has been suggested to lead to a softer structure with less resistance to unfolding [88].

In view of the above considerations, we addressed the modeling of a dimer of dimers to study whether cysteine crosslinking would be geometrically possible (Figure 4D). For this purpose, the vimentin dimer Model B, with the cysteine residue in thiol form, was used following protein-protein docking approaches. Several dimer-dimer assemblies were predicted and analyzed, and finally only two tetrameric complexes were selected that accomplished two conditions: (1) they presented an antiparallel orientation, according to the literature [17,22], and (2) the dimer backbones adopted an overall longitudinal alignment. These two "dimers of dimers" differed in the distance between cysteine residues: in the so-called "distant-CYS" arrangement, the cysteine residues were $22 \AA$ apart (Figure 4D, two upper panels), whereas in the "nearby-CYS" arrangement, the distance between cysteine residues was $4 \AA$ (Figure $4 \mathrm{D}$, two lower panels). The stability of these two tetrameric complexes was then studied by running 100 ns MD simulations (Figure S7). The structure of the "distant-CYS" complex bent during the simulation, and the terminal regions of the dimers fell apart (Figure 4D, two upper panels). On the contrary, the "nearby-CYS" tetrameric structure remained stable during the simulation, and only subtle changes in the conformation and distance between dimers were observed (Figure 4D, two lower panels). Therefore, only the "nearby-CYS" arrangement was considered for further calculations, given the fact that the distance between the cysteine residues (around $4 \AA$ ) is compatible with the possibility of an oxidative or chemical crosslinking (distance $<6 \AA$, DBB average crosslinking distance, $4.88 \AA$, [50]). Figure $4 \mathrm{E}$ through $\mathrm{G}$ show additional views of the dimerization interface of the "nearby-CYS" dimer of dimers putatively generated under oxidative conditions. The two antiparallel chains appeared bound through a strong network of ionic interactions, e.g., between the side chains of Arg300 and Glu346*, Lys303 and Glu338*, Arg308-Gln323 and Asp331*, Asp331 and Arg308*-Gln323*, Glu338 and Lys303*, and Glu346 and Arg300* (Figure 4F,G). Nevertheless, our results do not exclude that both "nearby-CYS" and "distant-CYS" arrangements of the vimentin tetrameric complex and potentially other assemblies, including those proposed from amino group crosslinking studies, coexist (schematized in Figure 1). Vimentin filaments are generally considered to be formed by eight tetramers per section. Early oxidative crosslinking studies of heterooligomers of vimentin and GFAP or desmin proposed that dimers involved in crosslinking would be in a symmetrical "face to face" disposition, implying the establishment of a cysteine disulfide between monomers in the same orientation [86,87]. Additionally, it was proposed that oxidative crosslinking was always intra-filament. However, later crosslinking studies using amine-reactive reagents have indicated that neighboring dimers within a filament are always antiparallel [22], and this has been reflected in several models [15,17,22] (see scheme in Figure 1). Therefore, several possible arrangements or orientations for the establishment of disulfide-bonded vimentin dimers may exist, although the consideration of all of them falls outside the scope of this study. Importantly, the coexistence of several oligomeric forms of soluble vimentin implies the presence of several subpopulations of Cys residues within different chemical environments; therefore, from the chemical viewpoint, their thiol groups would not be equivalent. Observation of the models of the dimer and the dimer of dimers can shed light on the oligomerization-dependent factors influencing the $\mathrm{pK}_{\mathrm{a}}$ of the cysteine residues. In the dimer model, thiol groups appear oriented outwards and are more accessible and surrounded by the solvent. In contrast, in addition to external thiol groups, the dimer of dimers presents thiol groups facing the dimerization interface. These internal Cys-SH groups are buried by the vicinal side chains. Moreover, one of these internal thiol groups establishes a hydrogen bond with the Gln324 CO group, which may favor a putative decrease of its $\mathrm{pK}_{\mathrm{a}}$, as has been described for peroxiredoxin active site Cys [89]. 
A

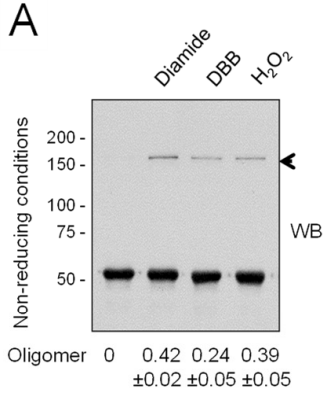

D

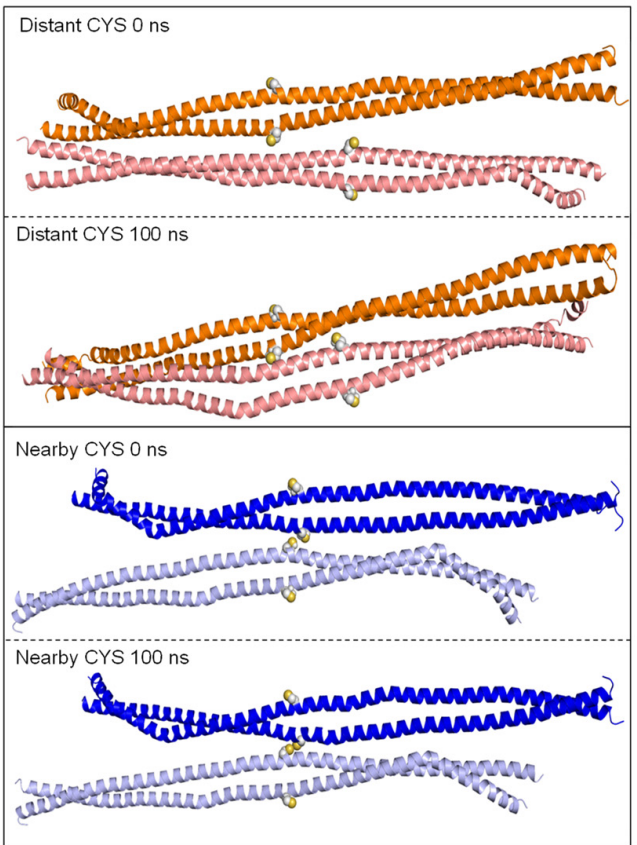

C
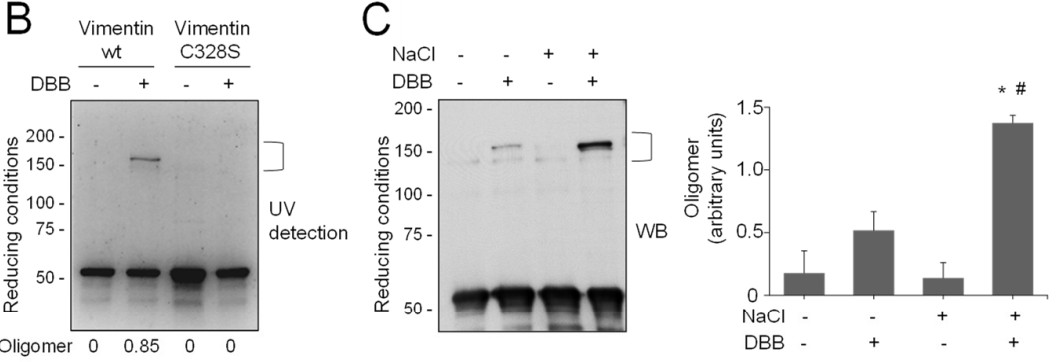

E

$\mathrm{F}$
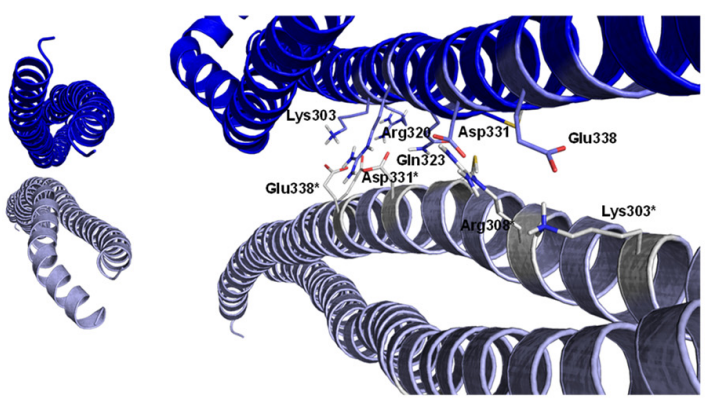

G

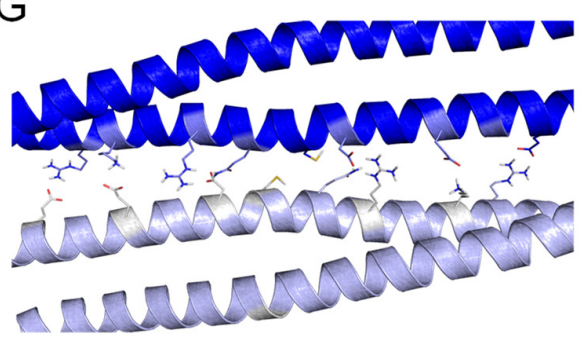

Figure 4. Cysteine-crosslinking of vimentin and potential conformations of the resulting dimers of dimers obtained by molecular dynamics. (A) Cysteine-crosslinking of vimentin $(4.7 \mu \mathrm{M}$ final concentration) upon incubation with vehicle, $1 \mathrm{mM}$ diamide, $24 \mu \mathrm{M} \mathrm{DBB}$, or $1 \mathrm{mM} \mathrm{H}_{2} \mathrm{O}_{2}$ for $1 \mathrm{~h}$ at r.t. Incubation mixtures were analyzed by SDS-PAGE under nonreducing conditions and western blot. The position of the oligomer detected under these conditions is marked by an arrow. The oligomer/monomer ratios are displayed below the plot as average values \pm SEM of three independent experiments. (B) Cys328 is required for DBB crosslinking. Vimentin wt or Cys328Ser ( $4.5 \mu \mathrm{M}$ final concentration) were incubated with $50 \mu \mathrm{M}$ DBB for $1 \mathrm{~h}$ at r.t, and subjected to SDS-PAGE, followed by Sypro Ruby staining for detection of vimentin monomeric and oligomeric (marked by a bracket) species under UV light. Cysteine-crosslinked oligomeric species were estimated by image scanning, and values are presented below the plot in arbitrary units. (C) Effect of NaCl-induced polymerization on DBB vimentin crosslinking. Vimentin wt ( $3.8 \mu \mathrm{M}$ final concentration) was incubated with vehicle or $150 \mathrm{mM} \mathrm{NaCl}$ for $10 \mathrm{~min}$ at $37^{\circ} \mathrm{C}$ (conditions that induce polymerization) and subsequently incubated for $1 \mathrm{~h}$ at r.t. with vehicle or $24 \mu \mathrm{M}$ DBB. Samples were analyzed by SDS-PAGE and western blot, and representative results are presented. The proportion of oligomeric (bracket) versus monomeric species is shown in the graph on the right. Results are average values of three assays \pm SEM. ${ }^{*} p<0.05$ vs. control (-NaCl, -DBB); $\# p<0.05$ vs. (-NaCl, $\left.+\mathrm{DBB}\right)$ by paired Student's $t$-test. (D) Models of vimentin dimers of dimers. Distant-CYS and nearby-CYS tetramers are represented in orange and blue, respectively. For each dimer, $100 \mathrm{~ns}$ of MD simulation were performed. The structures of the initial and last frames of the simulation are shown. Cys328 is represented in spheres. (E) Front view of the dimerization interface between both vimentin dimers in the nearby-CYS tetramer. (F,G) Semi-lateral (F) and lateral (G) views of both vimentin dimers in the nearby-CYS tetrameric association with details of the interactions that take place at the dimerization interface. Interacting residues are depicted as sticks. 


\subsection{Modeling the Interaction of the "Nearby-CYS" Vimentin Tetrameric Complex with Zinc}

Next, the interaction of the putative "nearby-CYS" tetrameric arrangement with zinc ions was studied (Figure 5). To this end, 100 zinc atoms were introduced, following the protocol described for the dimer, in order to fill all the possible zinc binding sites, and the systems were submitted to $30 \mathrm{~ns}$ MD simulations (Figure S8). A total of 26 zinc atoms remained in contact with vimentin residues more than $90 \%$ of the simulation time, confirming the existence of putative zinc hot spots in the vimentin "nearby CYS" tetrameric complex, which in fact double the number estimated for the vimentin dimer.
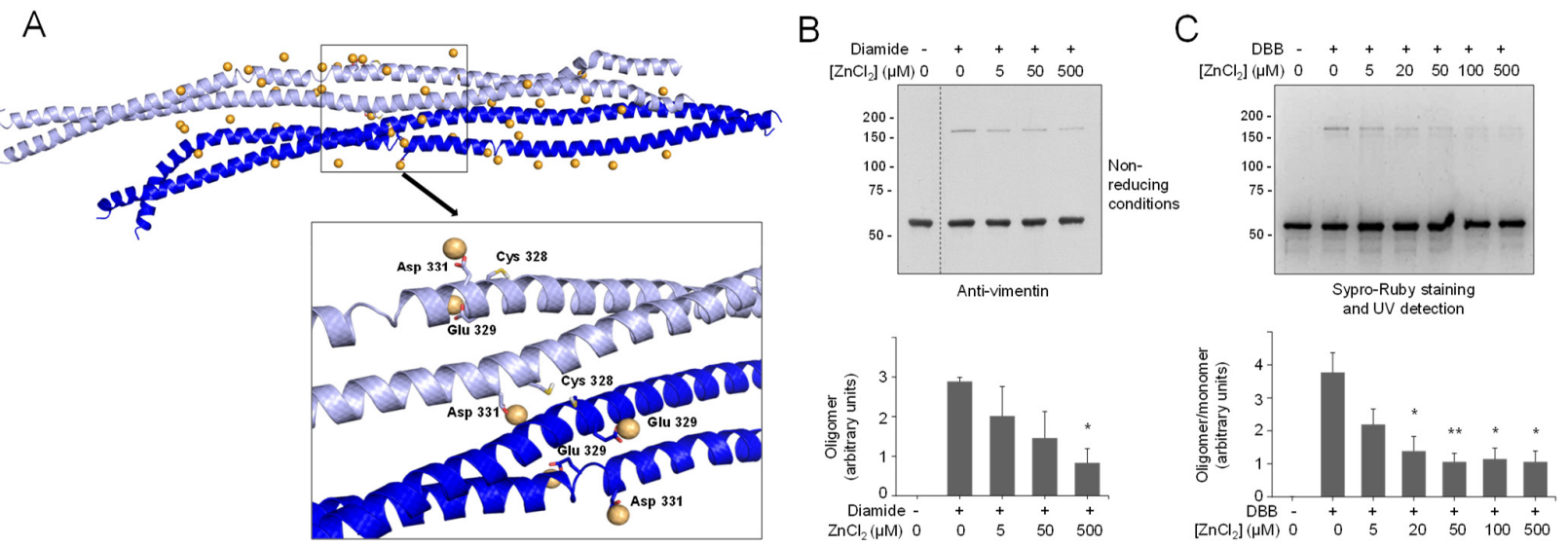

Figure 5. Interaction of zinc with the vimentin nearby-CYS vimentin tetrameric association and effect on cysteine crosslinking. (A) Zinc atoms remaining in the proximity of the vimentin nearby-CYS tetrameric association, with Cys 328 in its thiol form, are shown after $30 \mathrm{~ns}$ of MD simulation. Details of zinc interactions with residues in the vicinity of Cys328 are depicted in the enlarged image. (B,C) Effect of zinc on vimentin cysteine crosslinking. Vimentin wt (4.3 $\mu \mathrm{M}$ final concentration) was incubated in the presence of increasing concentrations of $\mathrm{ZnCl}_{2}$ for $1 \mathrm{~h}$ at r.t before induction of cysteine crosslinking by oxidation with $1 \mathrm{mM}$ diamide (B) or chemical crosslinking with $24 \mu \mathrm{M} \mathrm{DBB}(\mathbf{C})$, for an additional hour. Incubation mixtures were analyzed by non-reducing SDS-PAGE followed by western blot with anti-vimentin (B) or by SDS-PAGE followed by Sypro Ruby staining and UV detection $(C)$. The ratios of oligomeric vs. monomeric species are depicted in the graphs as average values \pm SEM from three assays. ${ }^{*} p<0.05$ vs. diamide or DBB in the absence of $\mathrm{ZnCl}_{2} ;{ }^{* *} p<0.001$ vs. DBB in the absence of $\mathrm{ZnCl}_{2}$, by unpaired Student's $t$-test.

A closer look at the region surrounding Cys328 showed stable interactions of zinc ions with the vicinal Glu329 and Asp331 residues, resembling those observed in the thiol-form of the dimer (Figure 5A).

Interactions equivalent to those found in the thiolate form of the dimer can also be deduced, as well as for several combinations of cysteine species (thiol or thiolate) that could occur. Therefore, we cannot exclude other dimer-dimer association patterns involving thiol and thiolate species of vimentin simultaneously, in which the coordination of zinc ions will occur through the predominant participation of the carboxylate or the cysteine residues, respectively. In fact, zinc atoms are observed to interact at the interface between both dimers. As more detailed structural information on vimentin filaments becomes available, it will be possible to predict whether zinc atoms participate in protein-protein interactions at the dimer or tetramer levels under physiological conditions or even in filament bundling, as suggested by recent evidence [44,45].

Next, the Cys328Ser dimer of dimers was constructed as a control. This structure revealed a significant difference with the wt tetrameric assembly consisting in the formation of a $\mathrm{H}$-bond between the $\mathrm{OH}$ groups of Ser328 residues from the different dimers, since their side chains fall in the correct orientation and distance (Figure S9). This contrasts with the wt tetrameric assembly, in which the distance between $\mathrm{SH}$ groups was longer and oscillated considerably along the MD simulation time. Mutation of the modelled Ser328 back to Cys328 led to a geometry similar to that of the wt, where the distance between $\mathrm{SH}$ groups does not correspond to a H-bond distance. Nevertheless, a Cys328Ser vimentin 
mutant will not undergo cysteine oxidative or chemical crosslinking for which it is not known whether this kind of association would occur.

\subsection{Zinc Protects Vimentin from Cysteine-Mediated Dimerization}

Since preincubation with zinc protects vimentin from modification by several electrophilic agents, we explored its effect on cysteine crosslinking. As shown in Figure 5B, diamide-induced disulfide oligomerization of vimentin was diminished by preincubation with $\mathrm{ZnCl}_{2}$ in a concentration-dependent manner. Moreover, crosslinking by DBB was markedly attenuated in the presence of zinc (Figure 5C). Importantly, this protective effect could be observed at low micromolar $\mathrm{ZnCl}_{2}$ concentrations that are similar to those of the protein present in the assay and in the range of the concentrations of zinc found in biological settings, e.g., in plasma [78]. Interestingly, the protective effect of zinc against cysteine modification and crosslinking affected not only soluble vimentin but also vimentin filaments preformed by addition of $\mathrm{NaCl}$ (Figure S10A,B).

\subsection{The Protective Effect of Zinc on Vimentin Is Selective}

Next, we explored the protective effect of zinc on vimentin crosslinking by other agents. For this purpose, we first used bifunctional cysteine crosslinkers differing in the length of their spacer arms (Figure S11). Interestingly, the protective effect of zinc appeared to inversely correlate with the crosslinkage length, resulting in a $76 \%$ inhibition of oligomer formation by DBB ( $4.88 \AA$ ), $56 \%$ inhibition of crosslinking by tris(2-maleimidoethyl)amine (TMEA, $10.3 \AA$ ), and no significant decrease in bismaleimidohexane-induced crosslinking (BMH, 13.0 A) (Figure 6A).

These results could suggest that interaction with zinc selectively protects a region of vimentin where cysteine residues from different tetramers or subunits are located at a relatively shorter distance, i.e., $<6 \AA$, than that measured in the "nearby-CYS" model. Of note, although TMEA is a three arm crosslinker, only vimentin dimers were observed. These results can be interpreted in several ways. First, as detailed above, the side chains of cysteine residues in the vimentin coiled-coil dimer would be oriented outwards. Therefore, crosslinking should involve monomers belonging to different dimers or tetramers. In this scenario, the possibility exists that zinc interaction in the proximity of Cys328 sterically precludes DBB crosslinking. Alternatively, zinc interaction with vimentin at this or other sites could induce conformational changes or associations in which cysteine residues are no longer at distances or orientations amenable to crosslinking with DBB or TMEA, while remaining susceptible to BMH-induced crosslinking. Indeed, micromolar zinc induces the association of soluble vimentin into distinct oligomeric assemblies that differ from ULF in their narrower dimensions and in their compromised elongation upon addition of $\mathrm{NaCl}$ [45]. In contrast, when interacting with preformed vimentin filaments, zinc promotes thickening and bundling, which are dependent on the presence of the cysteine residue, without altering filament integrity [45]. Therefore, these observations indicate that zinc does indeed induce some rearrangement of vimentin structures, at least in part by interacting with Cys328. Nevertheless, the fact that the protective effects of zinc occur both in soluble and filamentous vimentin (Figure S10A,B) suggests that they are not simply due to a tighter packing of the subunits. Moreover, both zinc-induced vimentin oligomerization [45] and protection from cysteine modification are reversible upon the addition of zinc chelators (Figure S10C), which indicates that the rearrangements do not imply a denaturation of the protein.

As stated above, the products of the crosslinking of vimentin through amino groups have been exhaustively studied, both in soluble vimentin and in filaments. This has led to the definition of the modes of assembly of vimentin tetramers and their position in filaments, schematized in Figure 1A [22,64,65]. In order to get further insight into the specificity of zinc protection, its effect on amine-crosslinking was explored. As previously reported, crosslinking with the bifunctional amine crosslinker disuccinimidyl tartrate (DST, spacer arm, $6.4 \AA$ ) induced the appearance of multiple vimentin oligomeric species (Figure $6 \mathrm{~B}$, 
left panel). Interestingly, preincubation with $\mathrm{ZnCl}_{2}$ did not diminish DST-induced vimentin crosslinking significantly or alter the pattern of oligomeric bands detected, either in the absence or presence of $\mathrm{NaCl}$ (Figure 6B, middle panel), suggesting that the protective effect of zinc is selective for thiol crosslinkers of a given length.
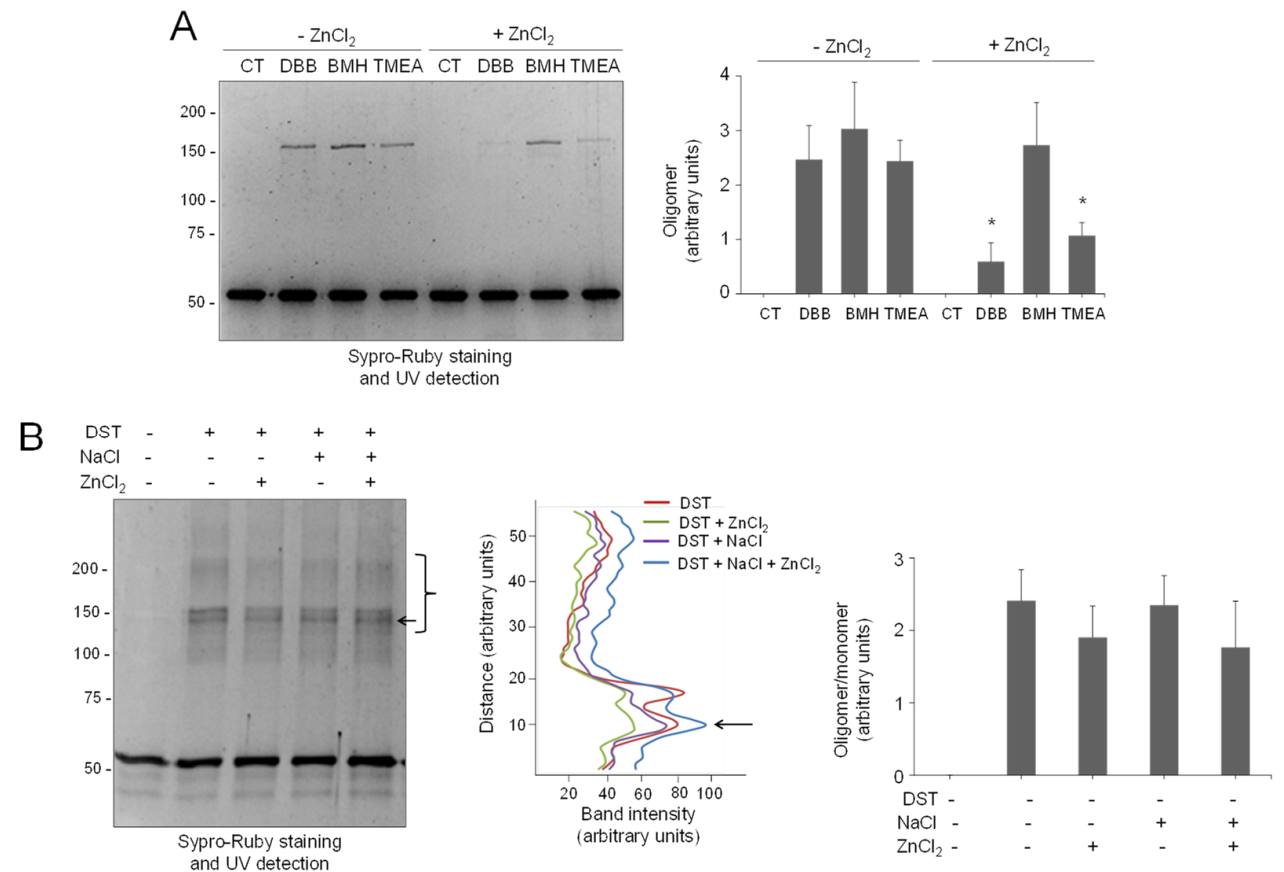

Figure 6. Selectivity of the protective effect of zinc on vimentin crosslinking. (A) Vimentin wt (4.3 $\mu \mathrm{M}$ final concentration) was incubated with vehicle or $500 \mu \mathrm{M} \mathrm{ZnCl}_{2}$ for $1 \mathrm{~h}$ at r.t., before the addition of the vehicle (DMSO) or the indicated cysteine crosslinkers at $24 \mu \mathrm{M}$ for incubation during $1 \mathrm{~h}$ more. Incubation mixtures were analyzed by SDS-PAGE and Sypro Ruby staining. Oligomeric species were estimated by image scanning, and mean values \pm SEM from three assays are represented in the graphs. ${ }^{*} p<0.05$, with respect to the reaction with the same crosslinker in the absence of $\mathrm{ZnCl}_{2}$ by paired Student's $t$-test. (B) Effect of zinc on vimentin crosslinking by an amine-reactive compound. Left panel, vimentin wt (5.8 $\mu \mathrm{M}$ final concentration), was incubated with vehicle, $150 \mathrm{mM} \mathrm{NaCl}$, or $500 \mu \mathrm{M} \mathrm{ZnCl} 2$ for $1 \mathrm{~h}$ at r.t. Incubation mixtures were then treated with the amine-reactive crosslinker disuccinimidyl tartrate (DST) at $400 \mu \mathrm{M}$ for $1 \mathrm{~h}$ at $37^{\circ} \mathrm{C}$ and analyzed as in (A). Middle panel, the intensity of the oligomeric species in the region delimited by the bracket was estimated and is depicted in the graph as a function of the distance (from bottom to top of the marked region). The arrow pointing to the most intense band is shown for reference. Right panel, the ratio of oligomeric (bracket) versus monomeric species was estimated and is depicted in the graph as mean values \pm SEM from three assays. No significant differences were found by paired Student's $t$-test.

\subsection{Interaction of Vimentin with Magnesium}

In contrast with the recently explored vimentin-zinc interaction $[9,90]$, the interaction of vimentin with other divalent cations, including $\mathrm{Ca}^{2+}$ and $\mathrm{Mg}^{2+}$, has been extensively studied [42,91]. In particular, magnesium has been reported to induce an electrostatic crosslinking of the vimentin tail segments, which impacts on the physical properties of vimentin filaments $[43,92]$. Therefore, we were interested in assessing the potential binding of magnesium in the area surrounding Cys328. MD simulations of the thiol form of the vimentin dimer in the presence of magnesium ions revealed that, in contrast with the two zinc ions bound in the proximity of the Glu329 and Asp331 hot spot, only one magnesium ion could bind to this region (Figure 7A and Figure S12). This fact could be due to the bigger size of the magnesium ion that would preclude a proper anchorage at the zinc ion site. Indeed, the binding of magnesium to vimentin has been reported to occur mainly at the C-terminal segment of the protein [42]. Thus, although vimentin behaves as a polyelectrolyte able to bind multiple divalent cations, our results indicate that their mode 
of binding and effects on the properties of the protein are not identical, with $\mathrm{Zn}^{2+}$ exerting distinctive effects in terms of concentration and, potentially, protein conformation [45].

A

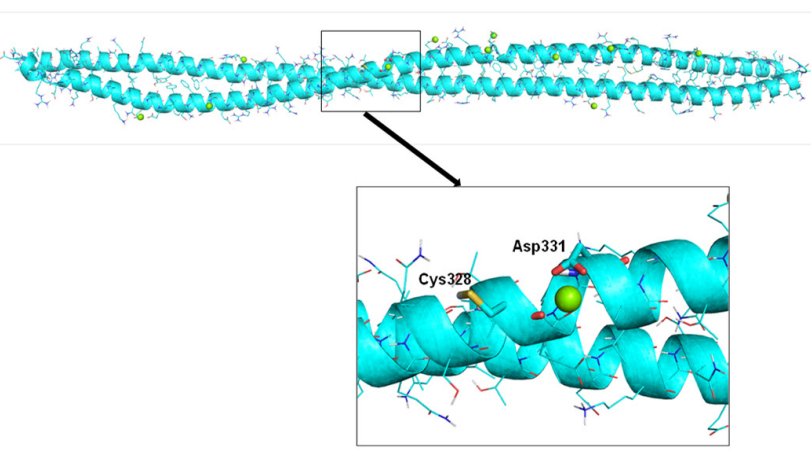

$\mathrm{B}$

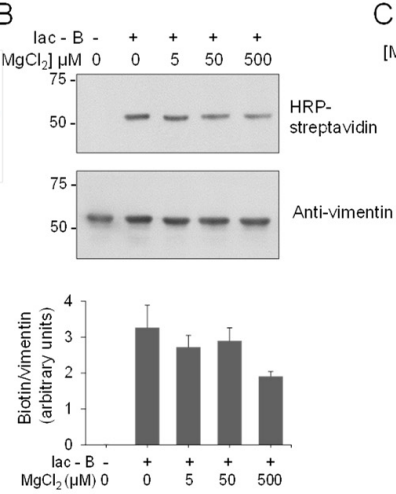

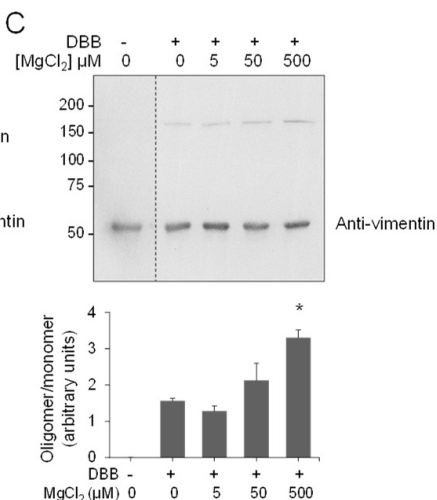

Figure 7. Interaction of magnesium with the vimentin dimer and effect on cysteine crosslinking. (A) Final snapshot of the MD simulation of vimentin dimer B in the presence of magnesium atoms (green spheres). The lower panel shows an enlarged view of the region surrounding Cys328. (B,C) Effect of magnesium on vimentin chemical modification by Iac-B (B) or crosslinking by DBB (C). Vimentin wt ( $4.3 \mu \mathrm{M}$ final concentration) was incubated with vehicle or $\mathrm{MgCl}_{2}$ at the indicated concentrations for $1 \mathrm{~h}$, at r.t, after which $10 \mu \mathrm{M}$ Iac-B (B) or $24 \mu \mathrm{M}$ DBB (C) were added for $30 \mathrm{~min}$ or $1 \mathrm{~h}$, respectively, at r.t. Graphs in the lower panels show mean values \pm SEM of at least three independent experiments. ${ }^{*} p<0.05$ vs. DBB by unpaired Student's $t$-test.

Next, we explored the potential protective effect of magnesium on vimentin cysteine alkylation and crosslinking. Low micromolar concentrations of $\mathrm{MgCl}_{2}$ did not diminish the incorporation of Iac-B into vimentin, although higher micromolar concentrations, i.e., $500 \mu \mathrm{M}$, tended to decrease Iac-B binding (Figure $7 \mathrm{~B}$ ). Moreover, $\mathrm{MgCl}_{2}$ did not exert any protective effect on DBB-induced vimentin crosslinking. Conversely, preincubation with $\mathrm{MgCl}_{2}$ at $500 \mu \mathrm{M}$ (Figure $7 \mathrm{C}$ ), or even at millimolar concentrations (Figure S13), that is, in a 100-1000-fold molar excess over the protein, increased the intensity of the DBB-induced dimer band. This could be related to the ability of millimolar magnesium to induce vimentin polymerization [43], which, as shown in Figure 4C for NaCl-induced polymerization, could facilitate DBB-induced vimentin crosslinking. Moreover, magnesium and calcium are known to mediate lateral interactions favoring filament attraction and aggregation [93], and hence they may favor conformations in which Cys328 from different tetramers or filaments become amenable to crosslinking by DBB.

In summary, based on our cysteine modification assays and computational models, we propose that the region surrounding the single cysteine residue of vimentin behaves as a hot spot for zinc binding, possibly modulating vimentin assembly. The proposal of this zinc-binding motif is compatible with the binding of this cation at multiple vimentin sites, and also with the potential contribution of distant segments of the protein, such as the head or tail domains, or of other dimers to zinc binding at the Cys328 region once the protein is organized in filaments. Indeed, results of the competition assay with the zinc chelator Zincon suggest the existence of several sites for zinc interaction in vimentin, with affinities in the nanomolar to micromolar range. These affinities would be clearly lower than those of some well-known zinc binding proteins including metallothionein or Keap1, which possess $K_{D}$ in the picomolar range $\left(10^{-11} \mathrm{M}\right)$ [62]. Nevertheless, besides high affinity sites, low affinity sites abound in cells or in the extracellular medium [94], including sites on certain zinc transporters or in proteins such as albumin or transferrin, for which $K_{D}$ in the nanomolar to micromolar range have been measured [60,62]. These low affinity carriers allow the cell to sense zinc deficiency early [95], in contrast to high affinity binding proteins, which will remain in their zinc-bound form in situations of mild zinc deficiency. Of note, within the intermediate filament family, keratins have long been known to bind zinc [96,97], although the precise site(s) of interaction, to the best of our knowledge, has not been 
elucidated. Moreover, Cys328 is not conserved in keratins. Interestingly, other cytoskeletal proteins, including actin and tubulin, can bind zinc and are considered zinc-scavenging proteins. In the case of tubulin, zinc can induce various assemblies, including microtubules, sheets, and macrotubes, depending on the experimental conditions. Remarkably, zinc appears to induce conformational changes affecting the lateral interactions of tubulin protofilaments, favoring the formation of sheets, in which protofilaments are assembled in an antiparallel fashion [98]. This associates with zinc-induced conformational changes affecting lateral contacts [99] through its interaction with histidine and glutamic residues from adjacent subunits [100]. Importantly, zinc availability affects tubulin thiol redox status and polymerization in neuronal cells [101]. Thus, in view of the current evidence, the cysteine hot spot for zinc binding in vimentin and putatively in other type III intermediate filament proteins could represent a selective site of interaction with potential implications for the regulation of these proteins by redox mechanisms, the study of which deserves further attention.

The pathophysiological consequences of the exposure of vimentin to zinc could be diverse depending on its location. We have previously shown that the vimentin network is thinner and more susceptible to oxidative damage in fibroblasts from patients with genetic zinc deficiency, i.e., acrodermatitis enteropathica [102], and these cellular alterations are ameliorated by supplementation with micromolar levels of zinc [9]. In addition, zinc is able to induce aggregation of vimentin and other proteins [45,103], which could contribute to pathologies associated with extracellular accumulations of vimentin and zinc, such as the pathological deposits that develop in diseases such as age-related macular degeneration [104]. Importantly, in a cellular context, zinc availability will influence vimentin dynamics by multiple mechanisms, including the control of enzymes modulating cellular redox status, zinc-dependent proteases, other cytoskeletal elements and/or transcription factors $[49,105]$.

\subsection{Concluding Remarks}

Compared to the other main cytoskeletal systems, microfilaments and microtubules, the regulation of the assembly and reorganization of intermediate filaments is insufficiently understood. Apart from posttranslational modifications, interaction of intermediate filaments with "cofactors", and specifically with divalent cations, is arising as a potential regulatory mechanism in vitro and in cells. In previous works, we identified the interaction of micromolar concentrations of zinc with vimentin and its impact on vimentin network organization and filament morphology, in vitro as well as in cellular models of disease. In addition, zinc, but not other divalent cations, affected the response of vimentin to oxidants and electrophiles, for which the presence of its single cysteine residue is an important factor. The findings herein reported provide a biochemical basis for the behavior of vimentin and, possibly, other members of the Type-III intermediate filament family, as redox sensors and potentially as drug targets, relying on the particular characteristics of the cysteine residue. Moreover, the identification of a hot spot for zinc binding in the region of the cysteine residue supports the role of zinc in vimentin organization and redox regulation and offers a working hypothesis to assess the role Type-III intermediate filaments in the pathogenesis of diseases associated with oxidative or electrophilic stress and/or zinc deficiency. Finally, given the central position of intermediate filaments in cytoskeletal crosstalk and in the homeostasis of cellular organelles, this interplay opens interesting avenues for the study of intermediate filaments as mediators of zinc availability and redox signaling, both in cells and in the extracellular medium, which has important implications in health and disease. 
Supplementary Materials: The following are available online at https: / www.mdpi.com/article/ 10.3390/antiox10071039/s1, Figure S1: MD simulations of vimentin dimer A (100 ns) and vimentin dimer B (200 ns). RMSD plots, Figure S2: MD simulation of vimentin dimer B with Zinc ions (30 ns). RMSD plot, Figure S3: MD simulation of vimentin dimer B with Cys328 thiol group as thiolate and in presence of Zinc ions (50 ns). RMSD plot, Figure S4: Determination of Cys328 pKa through various methods, Figure S5: Competition assay between vimentin and Zincon for zinc binding, Figure S6: MD simulation of vimentin dimer Cys328Ser mutant (50 ns). RMSD plot, Figure S7: MD simulations of vimentin tetrameric complexes (100 ns). (A) "distant-CYS" (Cys residues at a distance of $22 \AA$ ). (B) "nearby-CYS" (Cys residues at a distance of $4 \AA$ A). RMSD plots, Figure S8: MD simulation of vimentin tetrameric "nearby-CYS" complex with Zinc ions (100 ns). RMSD plot, Figure S9: MD simulation of vimentin tetrameric Cys328Ser mutant complex (100 ns). RMSD plot, Figure S10: Protective effect of zinc on Cys328 modification and crosslinking in soluble and polymerized vimentin, Figure S11: Cysteine crosslinkers used in this study, Figure S12: MD simulation of vimentin dimer B with Magnesium ions (100 ns). RMSD plot, Figure S13: Effect of millimolar magnesium concentration on the crosslinking of vimentin by DBB.

Author Contributions: Conceptualization, S.M.-S. and D.P.-S.; formal analysis, M.A.P.; funding acquisition, S.M.-S. and D.P.-S.; investigation, A.M., J.G.-C., M.A.P. and D.P.-S.; methodology, A.M. and J.G.-C.; supervision, S.M.-S. and D.P.-S.; writing-original draft, M.A.P., S.M.-S. and D.P.-S.; writing-review and editing, M.A.P. and D.P.-S. All authors have read and agreed to the published version of the manuscript.

Funding: This work was supported by the European Union's Horizon 2020 research and innovation program under the Marie Sklodowska-Curie Grant Agreement No. 675132 "Masstrplan", Grants SAF2015-68590-R and RTI2018-097624-B-I00 from Agencia Estatal de Investigación, MICINN/FEDER, Spain, and Instituto de Salud Carlos III/FEDER, RETIC ARADyAL RD16/0006/0021 to DPS; Grant CTQ2017-88353-R from MICINN to SMS; Grant BES-2015-071588 from MICINN to JGC.

Institutional Review Board Statement: Not applicable.

Informed Consent Statement: Not applicable.

Data Availability Statement: Data are contained within the article and supplementary material. Additional information can be obtained from the corresponding authors upon reasonable request.

Acknowledgments: We are indebted to MJ Carrasco for valuable technical assistance. Feedback from COST Actions CA15214 "EuroCellNet” and CA19105 "EpiLipidNet" is gratefully acknowledged.

Conflicts of Interest: The authors declare no conflict of interest.

\section{References}

1. Huber, F.; Boire, A.; Lopez, M.P.; Koenderink, G.H. Cytoskeletal crosstalk: When three different personalities team up. Curr. Opin. Cell. Biol. 2015, 32, 39-47. [CrossRef] [PubMed]

2. Duarte, S.; Viedma-Poyatos, A.; Navarro-Carrasco, E.; Martinez, A.E.; Pajares, M.A.; Perez-Sala, D. Vimentin filaments interact with the actin cortex in mitosis allowing normal cell division. Nat. Commun. 2019, 10, 4200. [CrossRef] [PubMed]

3. Latorre, E.; Kale, S.; Casares, L.; Gómez-González, M.; Uroz, M.; Valon, L.; Nair, R.V.; Garreta, E.; Montserrat, N.A.; Del Campo, A.; et al. Active superelasticity in three-dimensional epithelia of controlled shape. Nature 2018, 563, 203-208. [CrossRef] [PubMed]

4. Ramos, I.; Stamatakis, K.; Oeste, C.L.; Perez-Sala, D. Vimentin as a multifaceted player and potential therapeutic target in viral infections. Int. J. Mol. Sci. 2020, 21, 4675. [CrossRef]

5. Yu, M.B.; Guerra, J.; Firek, A.; Langridge, W.H.R. Extracellular vimentin modulates human dendritic cell activation. Mol. Immunol. 2018, 104, 37-46. [CrossRef]

6. Mendez, M.G.; Kojima, S.; Goldman, R.D. Vimentin induces changes in cell shape, motility, and adhesion during the epithelial to mesenchymal transition. FASEB J. 2010, 24, 1838-1851. [CrossRef]

7. Strouhalova, K.; Prechova, M.; Gandalovicova, A.; Brabek, J.; Gregor, M.; Rosel, D. Vimentin intermediate filaments as potential target for cancer treatment. Cancers 2020, 12, 184. [CrossRef]

8. Bollong, M.J.; Pietila, M.; Pearson, A.D.; Sarkar, T.R.; Ahmad, I.; Soundararajan, R.; Lyssiotis, C.A.; Mani, S.A.; Schultz, P.G.; Lairson, L.L. A vimentin binding small molecule leads to mitotic disruption in mesenchymal cancers. Proc. Natl. Acad. Sci. USA 2017, 114, E9903-E9912. [CrossRef]

9. Pérez-Sala, D.; Oeste, C.L.; Martínez, A.E.; Garzón, B.; Carrasco, M.J.; Cañada, F.J. Vimentin filament organization and stress sensing depend on its single cysteine residue and zinc binding. Nat. Commun. 2015, 6, 7287. [CrossRef] [PubMed] 
10. Mónico, A.; Duarte, S.; Pajares, M.A.; Pérez-Sala, D. Vimentin disruption by lipoxidation and electrophiles: Role of the cysteine residue and filament dynamics. Redox Biol. 2019, 23, 101098. [CrossRef]

11. Duarte, S.; Melo, T.; Domingues, R.; Alché, J.d.D.; Pérez-Sala, D. Insight into the cellular effects of nitrated phospholipids: Evidence for pleiotropic mechanisms of action. Free. Rad. Biol. Med. 2019, 144, 192-202. [CrossRef] [PubMed]

12. Helfand, B.T.; Chang, L.; Goldman, R.D. Intermediate filaments are dynamic and motile elements of cellular architecture. J. Cell Sci. 2004, 117, 133-141. [CrossRef] [PubMed]

13. Colakoglu, G.; Brown, A. Intermediate filaments exchange subunits along their length and elongate by end-to-end annealing. J. Cell Biol. 2009, 185, 769-777. [CrossRef]

14. Herrmann, H.; Haner, M.; Brettel, M.; Muller, S.A.; Goldie, K.N.; Fedtke, B.; Lustig, A.; Franke, W.W.; Aebi, U. Structure and assembly properties of the intermediate filament protein vimentin: The role of its head, rod and tail domains. J. Mol. Biol. 1996, 264, 933-953. [CrossRef]

15. Ando, S.; Nakao, K.; Gohara, R.; Takasaki, Y.; Suehiro, K.; Oishi, Y. Morphological analysis of glutaraldehyde-fixed vimentin intermediate filaments and assembly-intermediates by atomic force microscopy. Biochim. Biophys. Acta 2004, 1702, 53-65. [CrossRef]

16. Kreplak, L.; Richter, K.; Aebi, U.; Herrmann, H. Electron microscopy of intermediate filaments: Teaming up with atomic force and confocal laser scanning microscopy. Methods Cell Biol. 2008, 88, 273-297.

17. Sokolova, A.V.; Kreplak, L.; Wedig, T.; Mucke, N.; Svergun, D.I.; Herrmann, H.; Aebi, U.; Strelkov, S.V. Monitoring intermediate filament assembly by small-angle x-ray scattering reveals the molecular architecture of assembly intermediates. Proc. Natl. Acad. Sci. USA 2006, 103, 16206-16211. [CrossRef] [PubMed]

18. Saldanha, O.; Brennich, M.E.; Burghammer, M.; Herrmann, H.; Koster, S. The filament forming reactions of vimentin tetramers studied in a serial-inlet microflow device by small angle x-ray scattering. Biomicrofluidics 2016, 10, 024108. [CrossRef] [PubMed]

19. Winheim, S.; Hieb, A.R.; Silbermann, M.; Surmann, E.M.; Wedig, T.; Herrmann, H.; Langowski, J.; Mucke, N. Deconstructing the late phase of vimentin assembly by total internal reflection fluorescence microscopy (tirfm). PLoS ONE 2011, 6, e19202. [CrossRef]

20. Minin, A.A.; Moldaver, M.V. Intermediate vimentin filaments and their role in intracellular organelle distribution. Biochem. Biokhimiia 2008, 73, 1453-1466. [CrossRef] [PubMed]

21. Guzenko, D.; Chernyatina, A.A.; Strelkov, S.V. Crystallographic studies of intermediate filament proteins. Subcell. Biochem. 2017, $82,151-170$.

22. Steinert, P.M.; Marekov, L.N.; Parry, D.A. Diversity of intermediate filament structure. Evidence that the alignment of coiled-coil molecules in vimentin is different from that in keratin intermediate filaments. J. Biol. Chem. 1993, 268, 24916-24925. [CrossRef]

23. Mucke, N.; Wedig, T.; Burer, A.; Marekov, L.N.; Steinert, P.M.; Langowski, J.; Aebi, U.; Herrmann, H. Molecular and biophysical characterization of assembly-starter units of human vimentin. J. Mol. Biol. 2004, 340, 97-114. [CrossRef] [PubMed]

24. Premchandar, A.; Mucke, N.; Poznanski, J.; Wedig, T.; Kaus-Drobek, M.; Herrmann, H.; Dadlez, M. Structural dynamics of the vimentin coiled-coil contact regions involved in filament assembly as revealed by hydrogen-deuterium exchange. J. Biol. Chem. 2016, 291, 24931-24950. [CrossRef]

25. Aziz, A.; Hess, J.F.; Budamagunta, M.S.; FitzGerald, P.G.; Voss, J.C. Head and rod 1 interactions in vimentin: Identification of contact sites, structure, and changes with phosphorylation using site-directed spin labeling and electron paramagnetic resonance. J. Biol. Chem. 2009, 284, 7330-7338. [CrossRef] [PubMed]

26. Hess, J.F.; Budamagunta, M.S.; Aziz, A.; FitzGerald, P.G.; Voss, J.C. Electron paramagnetic resonance analysis of the vimentin tail domain reveals points of order in a largely disordered region and conformational adaptation upon filament assembly. Protein Sci. Publ. Protein Soc. 2013, 22, 47-55. [CrossRef]

27. Kirmse, R.; Qin, Z.; Weinert, C.M.; Hoenger, A.; Buehler, M.J.; Kreplak, L. Plasticity of intermediate filament subunits. PLoS ONE 2010, 5, e12115. [CrossRef]

28. Kelemen, G.H. Intermediate filaments supporting cell shape and growth in bacteria. In Procaryotic Cytoskeletons; Lowe, J., Amos, L.A., Eds.; Springer International Publishing: Cham, Switzerland, 2017; pp. 161-211.

29. Ahn, J.; Jo, I.; Kang, S.M.; Hong, S.; Kim, S.; Jeong, S.; Kim, Y.H.; Park, B.J.; Ha, N.C. Structural basis for lamin assembly at the molecular level. Nat. Commun. 2019, 10, 3757. [CrossRef]

30. Eldirany, S.A.; Lomakin, I.B.; Ho, M.; Bunick, C.G. Recent insight into intermediate filament structure. Curr. Opin. Cell Biol. 2021, 68, 132-143. [CrossRef]

31. Eldirany, S.A.; Ho, M.; Hinbest, A.J.; Lomakin, I.B.; Bunick, C.G. Human keratin 1/10-1b tetramer structures reveal a knob-pocket mechanism in intermediate filament assembly. EMBO J. 2019, 38, e100741. [CrossRef]

32. Chernyatina, A.A.; Guzenko, D.; Strelkov, S.V. Intermediate filament structure: The bottom-up approach. Curr. Opin. Cell Biol. 2015, 32, 65-72. [CrossRef] [PubMed]

33. Guzenko, D.; Strelkov, S.V. Ccfold: Rapid and accurate prediction of coiled-coil structures and application to modeling intermediate filaments. Bioinformatics 2017, 34, 215-222. [CrossRef]

34. Viedma-Poyatos, Á.; Pablo, Y.d.; Pekny, M.; Pérez-Sala, D. The cysteine residue of glial fibrillary acidic protein is a critical target for lipoxidation and required for efficient network organization. Free. Rad. Biol. Med. 2018, 120, 380-394. [CrossRef] [PubMed]

35. Duarte, S.; Viedma-Poyatos, A.; Mónico, A.; Pérez-Sala, D. The conserved cysteine residue of type iii intermediate filaments serves as a structural element and redox sensor. Free Rad. Biol. Med. 2018, 120, S84. [CrossRef] 
36. Bargagna-Mohan, P.; Hamza, A.; Kim, Y.E.; Khuan Abby Ho, Y.; Mor-Vaknin, N.; Wendschlag, N.; Liu, J.; Evans, R.M.; Markovitz, D.M.; Zhan, C.G.; et al. The tumor inhibitor and antiangiogenic agent withaferin a targets the intermediate filament protein vimentin. Chem. Biol. 2007, 14, 623-634. [CrossRef]

37. Burikhanov, R.; Sviripa, V.M.; Hebbar, N.; Zhang, W.; Layton, W.J.; Hamza, A.; Zhan, C.G.; Watt, D.S.; Liu, C.; Rangnekar, V.M. Arylquins target vimentin to trigger par-4 secretion for tumor cell apoptosis. Nat. Chem. Biol. 2014, 10, 924-926. [CrossRef]

38. Strelkov, S.V.; Herrmann, H.; Geisler, N.; Wedig, T.; Zimbelmann, R.; Aebi, U.; Burkhard, P. Conserved segments 1a and 2b of the intermediate filament dimer: Their atomic structures and role in filament assembly. EMBO J. 2002, 21, 1255-1266. [CrossRef]

39. Nicolet, S.; Herrmann, H.; Aebi, U.; Strelkov, S.V. Atomic structure of vimentin coil 2. J. Struct. Biol. 2010, 170, 369-376. [CrossRef]

40. Hess, J.F.; Voss, J.C.; FitzGerald, P.G. Real-time observation of coiled-coil domains and subunit assembly in intermediate filaments. J. Biol. Chem. 2002, 277, 35516-35522. [CrossRef]

41. Rogers, K.R.; Herrmann, H.; Franke, W.W. Characterization of disulfide crosslink formation of human vimentin at the dimer, tetramer, and intermediate filament levels. J. Struct. Biol. 1996, 117, 55-69. [CrossRef]

42. Lin, Y.C.; Broedersz, C.P.; Rowat, A.C.; Wedig, T.; Herrmann, H.; Mackintosh, F.C.; Weitz, D.A. Divalent cations crosslink vimentin intermediate filament tail domains to regulate network mechanics. J. Mol. Biol. 2010, 399, 637-644. [CrossRef]

43. Brennich, M.E.; Bauch, S.; Vainio, U.; Wedig, T.; Herrmann, H.; Koster, S. Impact of ion valency on the assembly of vimentin studied by quantitative small angle x-ray scattering. Soft Matter 2014, 10, 2059-2068. [CrossRef]

44. Wu, H.; Shen, Y.; Wang, D.; Herrmann, H.; Goldman, R.D.; Weitz, D.A. Effect of divalent cations on the structure and mechanics of vimentin intermediate filaments. Biophys. J. 2020, 119, 55-64. [CrossRef]

45. Mónico, A.; Zorrilla, S.; Rivas, G.; Pérez-Sala, D. Zinc differentially modulates the assembly of soluble and polymerized vimentin. Int. J. Mol. Sci. 2020, 21, 2426. [CrossRef] [PubMed]

46. Pérez-Sala, D.; Oeste, C.L.; Sánchez-Gómez, F.J. Vimentin gets a new glow from zinc. Oncotarget 2015, 6, 15742-15743. [CrossRef]

47. Alberts, I.L.; Nadassy, K.; Wodak, S.J. Analysis of zinc binding sites in protein crystal structures. Protein Sci. Publ. Protein Soc. 1998, 7, 1700-1716. [CrossRef]

48. Maret, W. Zinc coordination environments in proteins as redox sensors and signal transducers. Antioxid. Redox Signal. 2006, 8 , 1419-1441. [CrossRef]

49. Maret, W. The redox biology of redox-inert zinc ions. Free Radic Biol. Med. 2019, 134, 311-326. [CrossRef] [PubMed]

50. Green, N.S.; Reisler, E.; Houk, K.N. Quantitative evaluation of the lengths of homobifunctional protein cross-linking reagents used as molecular rulers. Protein Sci. Publ. Protein Soc. 2001, 10, 1293-1304. [CrossRef] [PubMed]

51. Schrodinger, LCC. The PyMol Molecular Graphics System. Version 1.8.

52. Berman, H.M.; Westbrook, J.; Feng, Z.; Gilliland, G.; Bhat, T.N.; Weissig, H.; Shindyalov, I.N.; Bourne, P.E. The protein data bank. Nucleic Acids Res 2000, 28, 235-242. [CrossRef] [PubMed]

53. Dominguez, C.; Boelens, R.; Bonvin, A.M. Haddock: A protein-protein docking approach based on biochemical or biophysical information. J. Am. Chem. Soc. 2003, 125, 1731-1737. [CrossRef]

54. Pierce, B.G.; Wiehe, K.; Hwang, H.; Kim, B.H.; Vreven, T.; Weng, Z. Zdock server: Interactive docking prediction of protein-protein complexes and symmetric multimers. Bioinformatics 2014, 30, 1771-1773. [CrossRef] [PubMed]

55. Sondergaard, C.R.; Olsson, M.H.; Rostkowski, M.; Jensen, J.H. Improved treatment of ligands and coupling effects in empirical calculation and rationalization of pka values. J. Chem. Theory Comput. 2011, 7, 2284-2295. [CrossRef]

56. Olsson, M.H.M.; Sondergard, C.R.; Rostkowski, M.; Jensen, J.H. Propka3: Consistent treatment of internal and surface residues inempirical pka predictions. J. Chem. Theory Comput. 2011, 7, 525-537. [CrossRef] [PubMed]

57. Herrmann, H.; Hofmann, I.; Franke, W.W. Identification of a nonapeptide motif in the vimentin head domain involved in intermediate filament assembly. J. Mol. Biol. 1992, 223, 637-650. [CrossRef]

58. Mónico, A.; Rodríguez-Senra, E.; Cañada, F.J.; Zorrilla, S.; Pérez-Sala, D. Drawbacks of dialysis procedures for removal of edta. PLoS ONE 2017, 12, e0169843. [CrossRef]

59. Krezel, A.; Maret, W. The biological inorganic chemistry of zinc ions. Arch Biochem. Biophys 2016, 611, 3-19. [CrossRef]

60. Parsons, D.S.; Hogstrand, C.; Maret, W. The c-terminal cytosolic domain of the human zinc transporter znt8 and its diabetes risk variant. FEBS J. 2018, 285, 1237-1250. [CrossRef]

61. Maret, W. New perspectives of zinc coordination environments in proteins. J. Inorg. Biochem. 2012, 111, 110-116. [CrossRef] [PubMed]

62. Kochanczyk, T.; Drozd, A.; Krezel, A. Relationship between the architecture of zinc coordination and zinc binding affinity in proteins-insights into zinc regulation. Met. Integr. Biometal Sci. 2015, 7, 244-257. [CrossRef]

63. Aziz, A.; Hess, J.F.; Budamagunta, M.S.; Voss, J.C.; Kuzin, A.P.; Huang, Y.J.; Xiao, R.; Montelione, G.T.; FitzGerald, P.G.; Hunt, J.F. The structure of vimentin linker 1 and rod $1 \mathrm{~b}$ domains characterized by site-directed spin-labeling electron paramagnetic resonance (sdsl-epr) and x-ray crystallography. J. Biol. Chem. 2012, 287, 28349-28361. [CrossRef] [PubMed]

64. Herrmann, H.; Aebi, U. Intermediate filaments: Molecular structure, assembly mechanism, and integration into functionally distinct intracellular scaffolds. Annu. Rev. Biochem. 2004, 73, 749-789. [CrossRef] [PubMed]

65. Parry, D.A.; Strelkov, S.V.; Burkhard, P.; Aebi, U.; Herrmann, H. Towards a molecular description of intermediate filament structure and assembly. Exp. Cell. Res. 2007, 313, 2204-2216. [CrossRef]

66. Song, C.; Weichbrodt, C.; Salnikov, E.S.; Dynowski, M.; Forsberg, B.O.; Bechinger, B.; Steinem, C.; de Groot, B.L.; Zachariae, U.; Zeth, K. Crystal structure and functional mechanism of a human antimicrobial membrane channel. Proc. Natl. Acad. Sci. USA 2013, 110, 4586-4591. [CrossRef] [PubMed] 
67. Fratelli, M.; Demol, H.; Puype, M.; Casagrande, S.; Eberini, I.; Salmona, M.; Bonetto, V.; Mengozzi, M.; Duffieux, F.; Miclet, E.; et al. Identification by redox proteomics of glutathionylated proteins in oxidatively stressed human $\mathrm{t}$ lymphocytes. Proc. Natl. Acad. Sci. USA 2002, 99, 3505-3510. [CrossRef]

68. Jia, J.; Arif, A.; Terenzi, F.; Willard, B.; Plow, E.F.; Hazen, S.L.; Fox, P.L. Target-selective protein s-nitrosylation by sequence motif recognition. Cell 2014, 159, 623-634. [CrossRef]

69. Kaschula, C.H.; Tuveri, R.; Ngarande, E.; Dzobo, K.; Barnett, C.; Kusza, D.A.; Graham, L.M.; Katz, A.A.; Rafudeen, M.S.; Parker, M.I.; et al. The garlic compound ajoene covalently binds vimentin, disrupts the vimentin network and exerts antimetastatic activity in cancer cells. BMC Cancer 2019, 19, 248. [CrossRef]

70. Roos, G.; Foloppe, N.; Messens, J. Understanding the pk(a) of redox cysteines: The key role of hydrogen bonding. Antioxid. Redox Signal. 2013, 18, 94-127. [CrossRef]

71. Sardi, F.; Manta, B.; Portillo-Ledesma, S.; Knoops, B.; Comini, M.A.; Ferrer-Sueta, G. Determination of acidity and nucleophilicity in thiols by reaction with monobromobimane and fluorescence detection. Anal. Biochem. 2013, 435, 74-82. [CrossRef]

72. Moutevelis, E.; Warwicker, J. Prediction of pka and redox properties in the thioredoxin superfamily. Protein Sci. Publ. Protein Soc. 2004, 13, 2744-2752. [CrossRef]

73. Lalmanach, G.; Saidi, A.; Bigot, P.; Chazeirat, T.; Lecaille, F.; Wartenberg, M. Regulation of the proteolytic activity of cysteine cathepsins by oxidants. Int. J. Mol. Sci. 2020, 21, 1944. [CrossRef] [PubMed]

74. Kooijman, M.; Bloemendal, M.; van Amerongen, H.; Traub, P.; van Grondelle, R. Characterization of multiple oligomeric vimentin intermediate filament units by transient electric birefringence measurements. J. Mol. Biol. 1994, 236, 1241-1249. [CrossRef]

75. Yuan, Y.; Knaggs, M.; Poole, L.; Fetrow, J.; Salsbury, F., Jr. Conformational and oligomeric effects on the cysteine pk(a) of tryparedoxin peroxidase. J. Biomol. Struct. Dyn. 2010, 28, 51-70. [CrossRef]

76. Viedma-Poyatos, A.; Pajares, M.A.; Pérez-Sala, D. Type iii intermediate filaments as targets and effectors of electrophiles and oxidants. Redox Biol. 2020, 36, 101582. [CrossRef] [PubMed]

77. Pace, N.J.; Weerapana, E. A competitive chemical-proteomic platform to identify zinc-binding cysteines. ACS Chem. Biol. 2014, 9 , 258-265. [CrossRef] [PubMed]

78. Goode, H.F.; Robertson, D.A.; Kelleher, J.; Walker, B.E. Effect of fasting, self-selected and isocaloric glucose and fat meals and intravenous feeding on plasma zinc concentrations. Ann. Clin. Biochem. 1991, 28, 442-445. [CrossRef] [PubMed]

79. Maret, W. Zinc and sulfur: A critical biological partnership. Biochemistry 2004, 43, 3301-3309. [CrossRef]

80. Fukunaka, A.; Fujitani, Y. Role of zinc homeostasis in the pathogenesis of diabetes and obesity. Int. J. Mol. Sci. 2018, 19, 476. [CrossRef]

81. Vinkenborg, J.L.; Nicolson, T.J.; Bellomo, E.A.; Koay, M.S.; Rutter, G.A.; Merkx, M. Genetically encoded fret sensors to monitor intracellular zn2+ homeostasis. Nat. Methods 2009, 6, 737-740. [CrossRef]

82. Cremers, C.M.; Jakob, U. Oxidant sensing by reversible disulfide bond formation. J. Biol. Chem. 2013, 288, 26489-26496. [CrossRef]

83. Ferrer-Sueta, G.; Manta, B.; Botti, H.; Radi, R.; Trujillo, M.; Denicola, A. Factors affecting protein thiol reactivity and specificity in peroxide reduction. Chem. Res. Toxicol. 2011, 24, 434-450. [CrossRef] [PubMed]

84. Lange, M.; Ok, K.; Shimberg, G.D.; Bursac, B.; Marko, L.; Ivanovic-Burmazovic, I.; Michel, S.L.J.; Filipovic, M.R. Direct zinc finger protein persulfidation by h2 s is facilitated by zn(2). Angew. Chem. Int. Ed. Engl. 2019, 58, 7997-8001. [CrossRef] [PubMed]

85. Sánchez-Gómez, F.J.; Díez-Dacal, B.; Pajares, M.A.; Llorca, O.; Pérez-Sala, D. Cyclopentenone prostaglandins with dienone structure promote cross-linking of the chemoresistance-inducing enzyme glutathione transferase p1-1. Mol. Pharm. 2010, 78, 723-733. [CrossRef] [PubMed]

86. Quinlan, R.A.; Franke, W.W. Molecular interactions in intermediate-sized filaments revealed by chemical cross-linking. Heteropolymers of vimentin and glial filament protein in cultured human glioma cells. Eur. J. Biochem. 1983, 132, 477-484. [CrossRef] [PubMed]

87. Quinlan, R.A.; Franke, W.W. Heteropolymer filaments of vimentin and desmin in vascular smooth muscle tissue and cultured baby hamster kidney cells demonstrated by chemical crosslinking. Proc. Natl. Acad. Sci. USA 1982, 79, 3452-3456. [CrossRef] [PubMed]

88. Ackbarow, T.; Buehler, M.J. Molecular mechanics of stutter defects in vimentin intermediate filaments. Exp. Mech. 2007, 49, 79-89. [CrossRef]

89. Poole, L.B. The basics of thiols and cysteines in redox biology and chemistry. Free Radic. Biol. Med. 2015, 80, 148-157. [CrossRef]

90. Mónico, A.; Zorrilla, S.; Pérez-Sala, D. Characterization of vimentin-zinc interaction and its impact on the response to electrophilic and oxidative stress. Free Rad. Biol. Med. 2017, 108, S46. [CrossRef]

91. Köster, S.; Lin, Y.-C.; Herrmann, H.; Weitz, D.A. Nanomechanics of vimentin intermediate filament networks. Soft Matter 2010, 6, 1910-1914. [CrossRef]

92. Dammann, C.; Noding, B.; Koster, S. Vimentin networks at tunable ion-concentration in microfluidic drops. Biomicrofluidics 2012, 6, 22009-2200910. [CrossRef]

93. Dammann, C.; Koster, S. Dynamics of counterion-induced attraction between vimentin filaments followed in microfluidic drops. Lab Chip 2014, 14, 2681-2687. [CrossRef]

94. Eide, D.J. Zinc transporters and the cellular trafficking of zinc. Biochim. Biophys. Acta 2006, 1763, 711-722. [CrossRef] [PubMed]

95. Bosdriesz, E.; Wortel, M.T.; Haanstra, J.R.; Wagner, M.J.; de la Torre Cortes, P.; Teusink, B. Low affinity uniporter carrier proteins can increase net substrate uptake rate by reducing efflux. Sci. Rep. 2018, 8, 5576. [CrossRef] [PubMed]

96. Fukuyama, K.; Murozuka, T.; Caldwell, R.; Epstein, W.L. Divalent cation stimulation of in vitro fibre assembly from epidermal keratin protein. J. Cell. Sci. 1978, 33, 255-263. [CrossRef] 
97. Mack, J.W.; Steven, A.C.; Steinert, P.M. The mechanism of interaction of filaggrin with intermediate filaments. The ionic zipper hypothesis. J. Mol. Biol. 1993, 232, 50-66. [CrossRef]

98. Nogales, E.; Wolf, S.G.; Downing, K.H. Structure of the alpha beta tubulin dimer by electron crystallography. Nature 1998, 391, 199-203. [CrossRef]

99. Nogales, E.; Whittaker, M.; Milligan, R.A.; Downing, K.H. High-resolution model of the microtubule. Cell 1999, 96, 79-88. [CrossRef]

100. Lowe, J.; Li, H.; Downing, K.H.; Nogales, E. Refined structure of alpha beta-tubulin at 3.5 a resolution. J. Mol. Biol. 2001, 313, 1045-1057. [CrossRef]

101. Mackenzie, G.G.; Salvador, G.A.; Romero, C.; Keen, C.L.; Oteiza, P.I. A deficit in zinc availability can cause alterations in tubulin thiol redox status in cultured neurons and in the developing fetal rat brain. Free Radic. Biol. Med. 2011, 51, 480-489. [CrossRef] [PubMed]

102. Vazquez, F.; Grider, A. The effect of the acrodermatitis enteropathica mutation on zinc uptake in human fibroblasts. Biol. Trace Elem. Res. 1995, 50, 109-117. [CrossRef] [PubMed]

103. Nan, R.; Farabella, I.; Schumacher, F.F.; Miller, A.; Gor, J.; Martin, A.C.; Jones, D.T.; Lengyel, I.; Perkins, S.J. Zinc binding to the tyr402 and his402 allotypes of complement factor h: Possible implications for age-related macular degeneration. J. Mol. Biol. 2011, 408, 714-735. [CrossRef] [PubMed]

104. Crabb, J.W.; Miyagi, M.; Gu, X.; Shadrach, K.; West, K.A.; Sakaguchi, H.; Kamei, M.; Hasan, A.; Yan, L.; Rayborn, M.E.; et al. Drusen proteome analysis: An approach to the etiology of age-related macular degeneration. Proc. Natl. Acad. Sci. USA 2002, 99, 14682-14687. [CrossRef] [PubMed]

105. Kaufman, Z.; Salvador, G.A.; Liu, X.; Oteiza, P.I. Zinc and the modulation of nrf2 in human neuroblastoma cells. Free Radic. Biol. Med. 2020, 155, 1-9. 